\title{
HEAT TRANSFER TO THE TRANSPIRED TURBULENT BOUNDARY LAYER
}

\author{
By
}

W. M. Kays

Report HMT-14

This study supported

by

The National Science Foundation

NSF GK-2201

and

The National Aeronautics and Space Administration

NGL 05-020-134

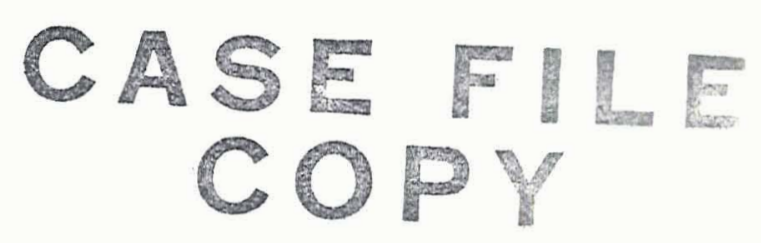

Thermosciences Division

Department of Mechanical Engineering

Stanford University

Stanford, California

June 1971 
HEAT TRANSFER TO THE TRANSPIRED TURBULENT BOUNDARY LAYER

By

W. M。 Kays

Report HMT-14

This study supported

by

The National Science Foundation

NSF GK-2201

and

The National Aeronautics and Space Administration

NGL $05-020-134$

Thermosciences Division

Department of Mechanical Engineering

Stanford University

Stanford,California

June 1971 


\section{Abstract}

This paper contains a summarization of five years work on an investigation on heat transfer to the transpired turbulent boundary layer. The experimental results are presented for friction coefficient and Stanton number over a wide range of blowing and suction for the case of constant free-stream velocity, holding constant certain blowing parameters. The problem of the accelerated turbulent boundary layer with transpiration is considered, experimental data are presented and discussed, and theoretical models for solution of the momentum equation under these conditions are presented. Data on turbulent. Prandt 1 number are presented so that solutions to the energy equation may be obtained. Some examples of boundary layer heat transfer and friction coefficient are presented using one of the models discussed, employing a finite difference solution method. 


\section{Nomenclature}

\section{English letter symbols:}

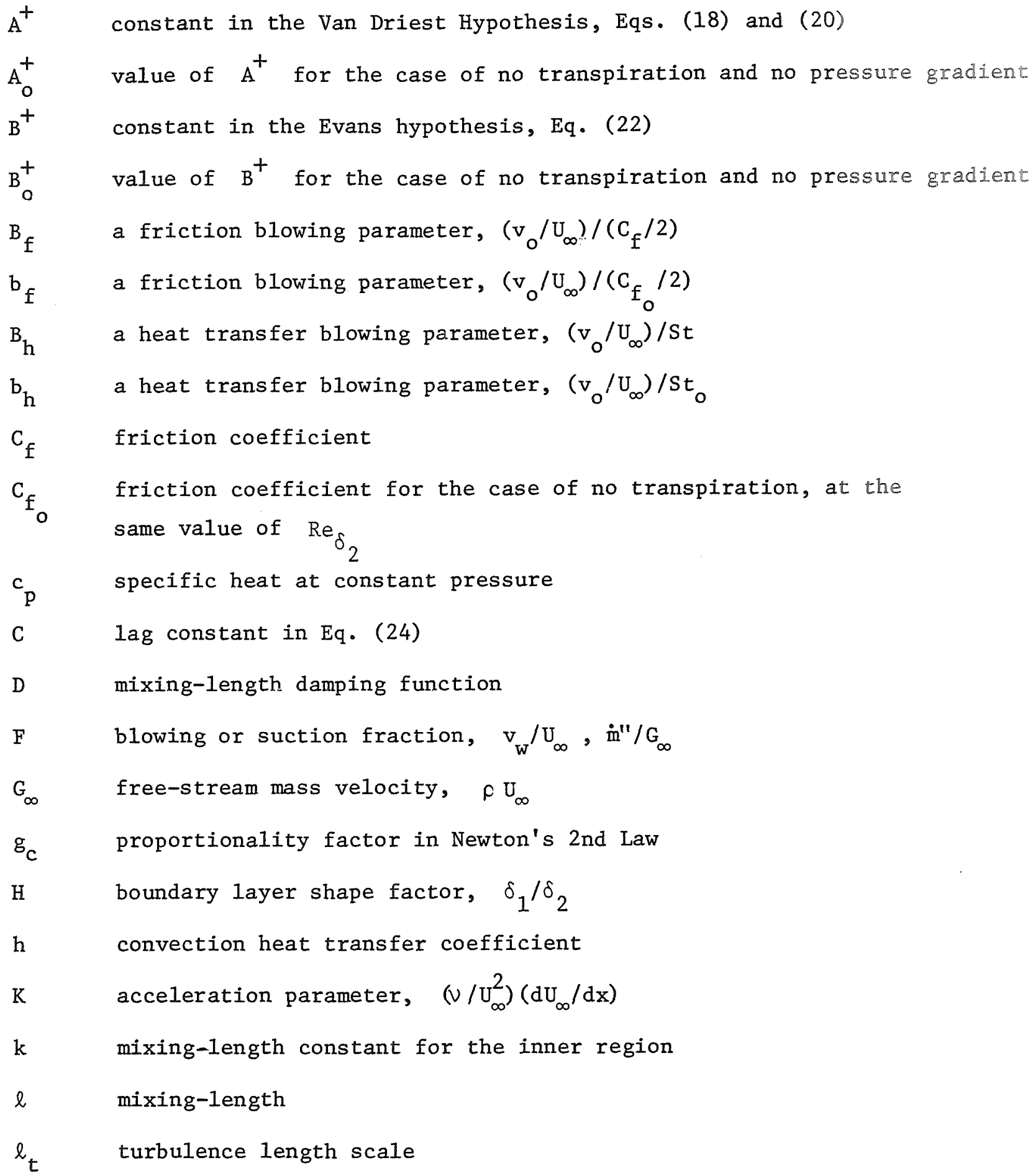




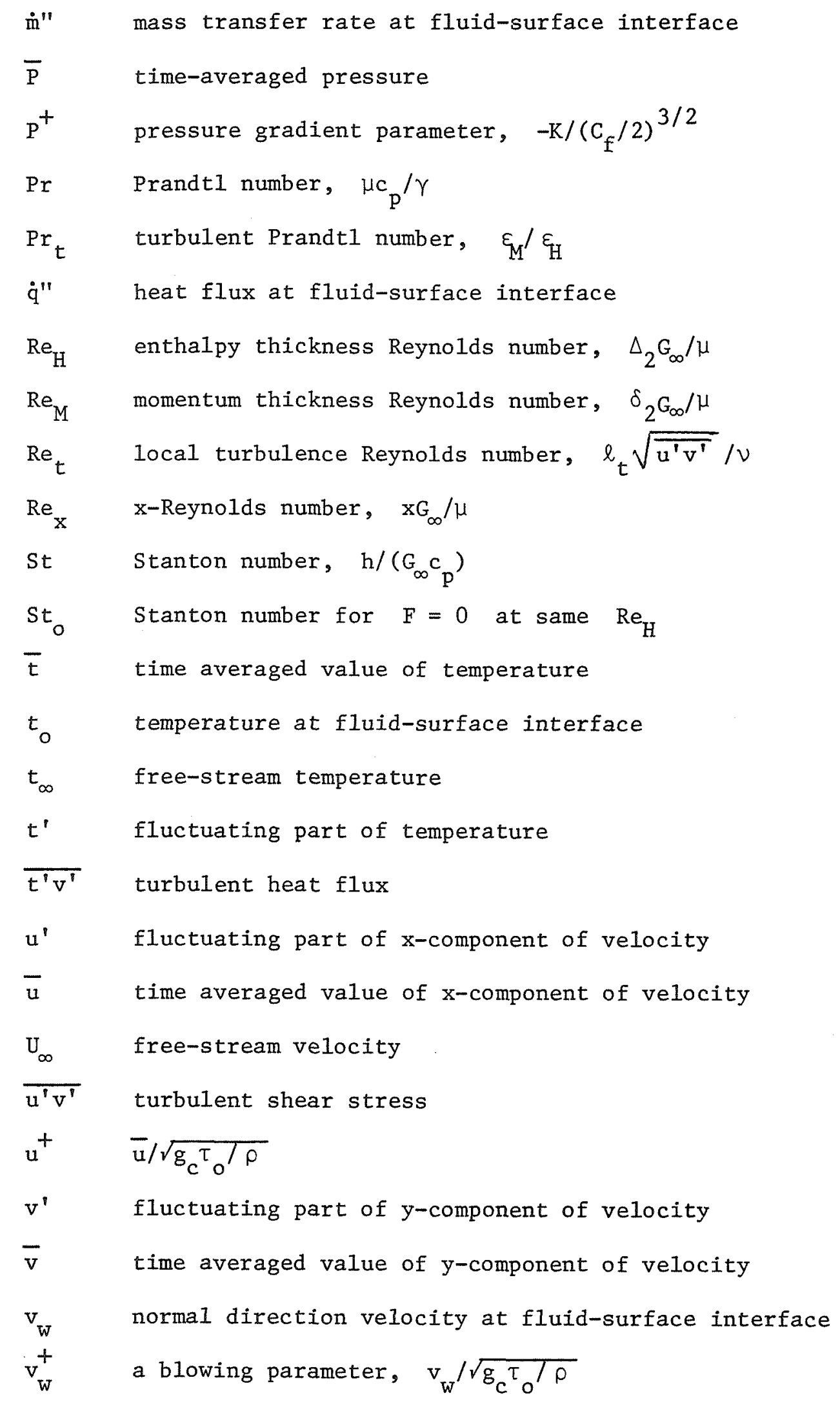




$$
\begin{array}{ll}
\mathrm{x} & \text { distance measured along surface in direction of flow } \\
\mathrm{x}^{+} & \mathrm{x} \sqrt{\mathrm{g}_{\mathrm{c}} \tau_{\mathrm{o}} / \rho / \nu} \\
\mathrm{y} & \text { distance measured normal to surface } \\
\mathrm{y}^{+} & \mathrm{y} \sqrt{\mathrm{g}_{\mathrm{c}} \mathrm{o}^{/ \rho}} / \mathrm{v} \\
\mathrm{y}_{\text {crit }}^{+} & \text {value of } \mathrm{y}^{+} \text {at edge of viscous sublayer }
\end{array}
$$

\section{Greek letter symbols:}

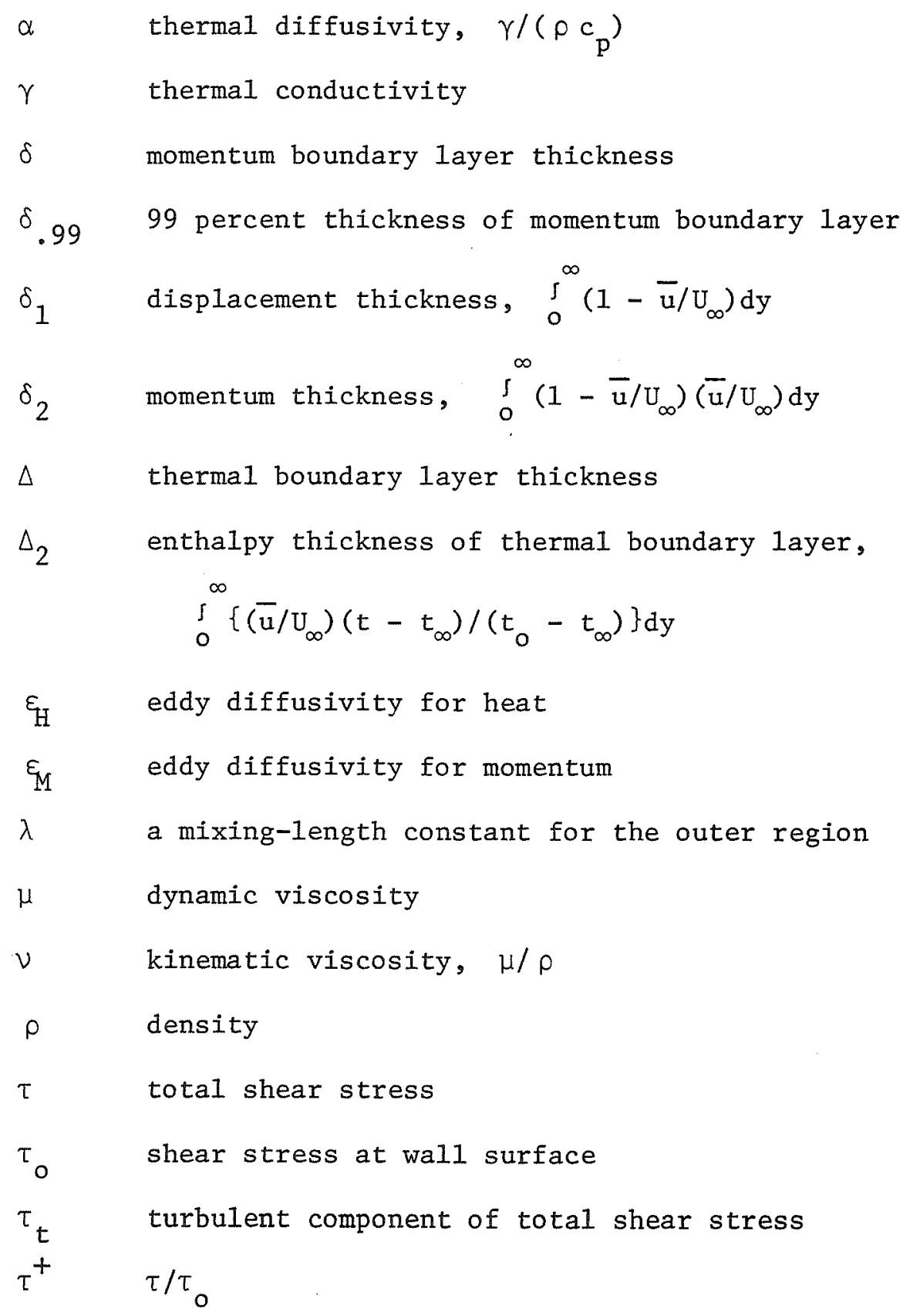


Introduction - Interest in the behavior of the transpired turbulent boundary layer extends back at least to the early 1950's when transpiration was first being investigated as a means of cooling aerodynamic surfaces under high velocity flight conditions. This interest is reflected in some of the pioneer papers and reports of Rubesin [1], and the paper of Dorrance and Dore [2]. While these contributions were on the theoretical side, experimental work began to take place, notably in the Chemical Engineering Department at M.I.I.s and is reflected in the papers of Mickley et al [3,4]. The early 1960's saw interest spreading with significant contributions from Great Britain, in. particular those of Stevenson [5], McQuaid [6], Black and Sarnecki [7], and contributions from the Soviet Union, for example Romanenko and Karchenko [8], and Kutateladse and Leont'ev [9]. During the past five years the number of contributions has grown into dozens. A complete bibliography, especialiy since 1960, is outside of the scope and purposes of the present paper, and the above citations are simply noted to provide some idea of interest intenm sity as a function of time.

Transpiration from a solid surface over which a fluid is flowing, and on which a boundary layer is developing, is of interest in a number of quite different types of applications. In the typical transpiration cooling application, the solid surface is constructed from sone kind of porous soilu nateriat. Cooling fluid, chemically the same as the free-stream, is then forced through the surface with the objective of protecting the surface from a hot free-stream. This is a boundary layer problem for which the normal component of velocity at the solid-fluid interface is non-zero, but otherwise the same momentum and energy boundary layer differential equations must be solved as for the 
non-transpired boundary layer. A variation on this problem occurs when the cooling fluid is a chemically different specie than the free-stream fluid. For example, helium might be injected as a coolant to protect a surface from a high temperature air free-stream. In this case the mass-diffusion equation of the boundary layer must be solved in addition to the momentum and energy equations. There are obviously similarities between these two types of problems, but also fundamental differences. Both are "mass transfer" problems in the sense that mass is transferred across the fluid-solid interface, but the latter is also a mass diffusion problem, while the former is not.

Another transpiration problem arises when there is evaporation or sublimation from an interface into a boundary layer, or condensation onto the interface. A further variation on the problem arises when there is chemical reaction either within the boundary layer or at the surface. In any of the cases cited, the direction of the flow normal to the surface at the interface could be into the surface, or it could be out of the surface. The terms "blowing" and "suction" are frequently used to denote the direction of flow at the interface, while the word "transpiration" generally is taken to embrace both cases. Suction is sometimes used as a scheme for aerodynamic boundary layer control because it is possible to inhibit or prevent boundary layer separation by suction.

These various types of applications suggest why chemical, mechanical and aeronautical engineers have all made significant contributions to the theory, and the terminology to a certain extent reflects these various origins of interest. 
In 1965, the author and his colleagues embarked on a comprehensive experimental investigation of heat transfer to the transpired turbulent boundary layer with the objectives of resolving some of the contradictions in the earlier data, filling in obvious holes, and ultimately of obtaining necessary information for a more fundamental theory so that adequate boundary layer predictions can be made over a wide variety of operating conditions. To date, this work has been entirely concerned with the single chemical component problem (i.e., no mass diffusion) using air as the working medium, and employing small temperature differences for the heat transfer work, so that it is effectively the constant property boundary layer that is considered. At the present time, the technical application that provides the major impetus for this program is the prospect of cooling gas turbine blades by transpiration. This application involves the transpired boundary layer with large temperature differences, as well as other complications that will be briefly discussed at the end of the paper, but a fundamental understanding of the behavior of the idealized low-velocity, constant property case is essential if any significant progress is going to be made in the more complex. applications.

The objective of this paper is to present a summary of some of the more significant results and conclusions that have resulted from this program. Much of what will be presented (although not al1) has appeared in various reports and papers, but significant portions of the work have now been completed, and it seems an appropriate time to attempt to summarize.

The general problem considered is perhaps best illustrated by reference to Fig. 1. Air flows at a steady rate along a flat surface which is porous, and through which air can be forced into the boundary layer or withdrawn from 
the boundary layer (blowing or suction). It is presumed that the surface is aerodynamically smooth, which means that the transpiration holes are small relative to the thickness of the laminar sublayer of the boundary layer, and that their spacing is small relative to the thickness of the laminar sublayer. It is presumed that the solid surface is a heat conductor, and that heat can be conducted to or from the surface. It is further presumed that the surface construction is such that the transpired fluid is in thermal equilibrium with the solid surface at the interface. In general, we would like to consider the case where the mass transfer rate $\mathrm{m}^{\prime \prime}$ (i.e., the transpiration rate) is any arbitrary function of distance $x$ along the surface, where the convection heat transfer rate $\dot{q}^{\prime \prime}$ is any arbitrary function of distance $x$ along the surface, and/or the surface temperature $t_{0}$ is any arbitrary function of $x$. We would like to consider the case where the free-stream velocity $U_{\infty}$ may vary in any arbitrary manner with $x$, but we will restrict the free-stream temperature $t_{\infty}$ to a constant. The boundary layer is two-dimensional, with the coordinate $y$ being used to measure the distance normal to the surface. Under these conditions, we are interested in the development of a momentum boundary layer, characterized by a thickness $\delta$, and a thermal boundary layer characterized by a thickness $\Delta$.

It should be added that the problem described, but with the boundary layer laminar rather than turbulent, is of equal interest in many applications including the turbine blade cooling one. However, a wealth of closed form solutions exist for particular idealized cases, and with modern finite-difference calculation methods the laminar problem poses no particular difficulty even for very complicated boundary conditions. 
The remainder of the paper is in six sections. The case of a constant free-stream velocity is considered first. Experimental results covering the entire range of blowing and suction are presented for the case of a constant rate of transpiration and a constant surface temperature. Methods of extending these results to problems of arbitrarily varying transpiration rate and arbitrarily varying surface temperature, using the integral equations of the boundary layer, are then discussed.

Next the problem of an accelerated free-stream velocity is considered. It will be seen that simple correlations together with the integral equations of the boundary layer no longer appear adequate as tools for performance prediction, and a more fundamental theory is needed.

The two sections following contain a discussion of some models that can be used for direct solution (by finite difference methods) of the momentum and energy differentiai equations of the boundary layer. Constants for use in these models, derived from experimental data, are presented.

Finally, some examples of predictions using one of the models applied to some very difficult cases are presented. The paper ends with a discussion of some of the future problems which must be considered. 
The Case of Constant Free-Stream Velocity - It is first instructive to examine the momentum integral equation of the boundary layer, particularized to the case of constant properties and constant free-stream velocity.

$$
\frac{\mathrm{d} \delta_{2}}{\mathrm{dx}}=\mathrm{C}_{\mathrm{f}} / 2+\mathrm{v}_{\mathrm{o}} / \mathrm{U}_{\infty}
$$

An alternative form is obtained if the friction coefficient is factored from the right-hand side, and if the resulting term containing the transpiration velocity $v_{0}$ is defined as a "blowing parameter", $B_{E}$.

$$
\frac{d \delta_{2}}{d x}=\left(c_{f} / 2\right)\left[1+\frac{v_{o} / U_{\infty}}{C_{f} / 2}\right]
$$

or

$$
\frac{d e_{M}}{d e_{X}}=\left(C_{f} / 2\right)\left(I+B_{f}\right)
$$

In laminar boundary layer theory, similarity solutions to the momentum equations are obtained when $B_{\text {ff }}$ is maintained constant along the surface. Since for a laminar boundary layer $C_{f} / 2$ varies as $\operatorname{Re}_{\mathrm{X}}^{-1 / 2}$, similar velocity profiles are then evidently obtained if the rate of blowing (or suction) decreases along the surface as the square root of distance $x$. For a turbulent boundary layer, an analogous situation obtains. $B_{f}$ can be interpreted physically as the ratio of the transpired momentum rate to the shear force. If this ratio is maintained constant along the surface, similar profiles are obtained for a laminar boundary layer, and for a turbulent boundary layer a so-called "equilibrium" boundary layer is obtained, one that possesses outer 
region similarity of velocity profiles. However, in the case of the turbulent boundary layer, $\mathrm{C}_{\mathrm{f}} / 2$ tends to vary with distance $\mathrm{x}$ appromimately as $x^{-0.2}$, so constant $B_{f}$ corresponds to a blowing rate, $v_{0} / U_{\infty}$, which decreases in the direction of flow as $x^{-0.2}$.

The constant $B_{f}$ boundary layer thus appears to be a fundamental case, and was a case studied by Simpson, et al [10]. Simpson alsc did extensive testing holding $v_{o} / J_{\infty}$ constant ratier than $B_{f}$ " (Hereafter $v_{0} / U_{\infty}$ will be referred to as $F$, the blowing fraction. Note also that since only" the constant density case is being discussed, $v_{0} / U_{\infty}=\dot{\mathrm{m}}^{\prime \prime} / G_{\infty}$, i.e., $F$ is a mass flux ratio, and this is how it should be interpreted in compressible flow applications.)

One of the conclusions from Simpson's work is that $\mathrm{C}_{f} / 2$ can be expressed as a function of the momentum thickness Reynolds number, $R e_{M}$, and that virtually the same function or relationship is obtained for experiments at constant $F$ as for experiments at constant $B_{f}$. In facts this function seems to be remarkably independent of how $F$ varies along the surface, and it is only for step changes in $F$ that any appreciable difference can be noted.

A further point of interest that can be seen in the momentum integral. equation, Eq. (1), is that when $v_{0} / U_{\infty}=F$ is negative, the possibility exists that the right-hand side of the equation will go to zero, leading to a situation where the momentum thickness of the boundary layer does not grow with $\mathrm{x}$. As a matter of fact, this is a situation that will always be approached where $F$ is maintained constant and negative. $C_{f} / 2$ has a decreasing tendency with increasing momentum thickness, starting indefinitely high at the beginning of the plate, and so regardless of the value of $F$ 
this point of equilibrium will ultimately be reached. This is commonly called the "asymptotic suction layer". Note that for this condition $B_{f}$ is equal to -1 , and thus -1 represents a lower limit on $B_{f}$. Simpson's experiments can be correlated within the limits of the experimental uncertainty of the data itself by the following equation.

$$
C_{f} / 2=0.0130 R e_{M}^{-.25}\left[\hat{\alpha n}\left(I+E_{f} j / B_{f}\right]^{0.77}\right.
$$

A plot of Eq. (4) is shown on Fig. 2. Since $\mathrm{C}_{f} / 2$ is contained within $\mathrm{B}_{\mathrm{f}}$, Eq. (4) is a little awkward to use. In Fig. 2, $\mathrm{F}$ is employed as a parameter as well as $B_{f}$, taking advantage of the experimental fact that the same functional relationship seems to be obtained for constant $F$ experiments as for constant $B_{f}$ experiments.

The fact that moving alorg a corstant $b_{f}$ line leacis to decreasing values of $F$ can be clearly seen. Note also that the lines for negative constant $F$ are slightly concave upwards. If extended, these lines will approach values of $\mathrm{C}_{\mathrm{f}} / 2$ equal to the negative of $\mathrm{F}$. An alternative way of presenting these results is shown in Fig. 3 where the ratio of $\mathrm{C}_{\mathrm{f}}$ to the value of $\mathrm{C}_{\mathrm{f}}$, at the same momentum thickness Reynolds number for the case of no transpiration, is plotted as a function of another blowing parameter, $b_{f} \cdot b_{f}$ differs from $B_{f}$ in that $F$ is divided by the value of $\mathrm{C}_{f} / 2$ for no transpiration, at the same momentum thickness Reynolds number, rather than with respect to the 1ocal value of $C_{f} / 2 \cdot b_{f}$ is thus a more convenient parameter to use in presenting data on $C_{f}$ because it does not contain the value of $C_{f}$ sought. Interestingly, Eq. (4) plots as a single line on this diagram, and illustrates 
vividly the influence of blowing and suction on the friction coefficient. Also superimposed on the diagram are the results of the laminar similarity solutions mentioned earlier. The behavior is quite analogous, especially on the suction side, but it should be noted that the laminar similarity solution does lead to a zero value of $C_{f}$ at $b_{f}=3.47$. This point is generally referred to as the "blow-off" point, and it is the value at which the boundary layer is literally blown off the wall. However, Eq. (4) only approaches zero and does not indicate a definite blow-off point. There is no reason to suppose that blow-off is not just as real a phenomena for the turbulent boundary layer as for a laminar boundary layer, but it is extremely difficult to detect experimentally because the uncertainty in measuring $C_{f}$ becomes indefinitely large as $C_{f}$ approaches zero. The fact that Eq. (4) does not indicate blow-off should only be interpreted as a deficiency in the form of the empirical equation used. A popular rule of thumb is that blow-off for a turbulent boundary layer will occur at approximately $F=0.01$, and this rule of thumb is certainly not inconsistent with the results on Fig. 3 when it is noted that the uncertainty in $\mathrm{C}_{f} / \mathrm{C}_{\mathrm{fo}}$ is of the order of magnitude 0.1 at the right-hand extreme of the diagram.

The shape factor of the boundary layer, $\mathrm{H}$, is often of interest, and the following equation is an empirical representation of Simpson's measurenents fur consint free-stream velocity.

$$
\mathrm{H}=1.0 /\left\{1.0-3.1 \sqrt{\mathrm{C}_{f} / 2}\left[\left(1+\mathrm{B}_{\mathrm{b}}\right)^{1 / 2}+\left(1+0.635 \mathrm{~B}_{f}\right)^{1 / 2}\right]\right\}
$$

A plot of Eq. (5) is shown on Fig. 4. The significant point is that blowing leads to high values of $H$, and that for $F$ approaching $0.01 \quad H$ 
becomes greater than 2.0. It is perhaps worth noting that for the nontranspired boundary layer in an adverse pressure gradient, separation generally tends to occur when $H$ is somewhere above 2.0. This is one of many instances of similarity between the behavior of the transpired flat-plate boundary layer with positive transpiration, and the adverse pressure gradient non-transpired boundary layer.

It is only fair to remark that the friction coefficient data of Simpson, upon which this entire discussion is based, while unquestionably the most extensive data available, has been the subject of some controversy. The case against it has been very well put by Squire [11], who feels that Simpson's friction coefficients are too high. There is ample room for argument because of the very considerable difficulty in measuring friction coefficients, and the different ways in which experimental data can be interpreted. The writer would only add at this time that friction coefficients close to Simpson's have been measured in subsequent work on the same apparatus, but in connection with accelerating flows, and where other methods of data interpretation have been used. Furthermore, there is an internal consistency between Simpson's data and the heat transfer data to be discussed shortly. There seems no question that the experimental uncertainty in the heat transfer data is less than for the friction coefficient data because the heat transfer rates are measured by a direct method. The internal consistency shows up particularly in the theoretical models to be discussed later; a single, reasonable model can be used to predict both the heat transfer data and the friction coefficient data equally well. 
Turning now to the case of heat transfer with transpiration, but with free-stream velocity constant and surface temperature constant, consider first the energy integral equation of the boundary layer, particularized to the case of constant properties, constant free-stream velocity, and constant surface temperature.

$$
\frac{d \Delta_{2}}{d x}=S t+v_{0} / U_{\infty}
$$

A heat transfer blowing parameter, $B_{h}$, is defined in a manner completely analogous to the definition of the friction blowing parameter.

$$
\begin{aligned}
& \frac{\mathrm{d} \Delta_{2}}{\mathrm{dx}}=S t\left[1+\frac{\mathrm{v} e^{/ \mathrm{U}_{\infty}}}{\mathrm{St}}\right] \\
& \frac{\mathrm{dRe} H}{\mathrm{dRe}}=\operatorname{st}\left(1+\mathrm{B}_{\mathrm{h}}\right)
\end{aligned}
$$

The blowing parameter $B_{h}$ can be interpreted physically as the ratio of the transpired thermal energy to the conducted thermal energy. Again, it is not surprising that holding this parameter constant along a surface leads to similarity of temperature profiles in the laminar boundary layer case, and apparently leads to outer region temperature profile similarity in the turbulent boundary layer case. There is also another physical significance to constant $B_{h}$. In the case of blowing, if the transpired fluid originates from a constant temperature source or plenum below the surface, and if there is no independent energy source within the surface wall 
itself, constant $B_{h}$ yields a constant surface temperature $t_{0}$. In other words, to obtain a constant surface temperature by transpiring a fluid which starts out at a single uniform temperature, it is necessary for the blowing fraction $F$ to decrease along the surface in such a manner as to hold $B_{h}$ constant. Experimentally it is found that $F$ must decrease as approximately the 0.2 power of $x$, as in the case of constant $B_{f} \cdot$ Note further that a constant negative value of $F$ must lead to an asymtotic suction layer for the same reasons as were discussed for the momentum boundary layer. In other words, under conditions of constant suction, Stanton number approaches a minimum, equal to the negative of $F$.

The experiments of Moffat and Kays [12], and of Whitten, et al [13], indicate that although $B_{h}$ probably represents the more fundamental case, constant $F$ yields to all intents and purposes the same value of stanton number for a given value of enthalpy thickness Reynolds number, $R e_{\mathrm{H}}$, and $F$. In fact, Whitten's results indicate that $F$ can vary rather considerably along the surface with negligible effect upon this relationship. The very extensive data of Moffat, and of Whitten, for uniform surface temperature; can be represented quite adequately by the following empirical equation.

$$
S t=0.0128 \operatorname{Pr}^{-0.5}\left[\ln \left(1+B_{h}\right) / B_{h}\right]^{1.25}\left[1+B_{h}\right]^{0.25}
$$

Equation (8) is plotted on Fig. 5 where both $B_{h}$ and the more convenient $F$ are used as parameters. It should be emphasized that strictly speaking these data apply only for cases where the virtual origin of both the thermal and momentum boundary layers is the same, which means that the 
thermal boundary layer thickness is of the same order magnitude as the momentum boundary layer thickness (actualiy about 10\% greater for $\operatorname{Pr}=0.7$ and $F=0$ )。 If the thermal boundary layer thickness is substantially different from the momentum boundary layer thickness, and this can occur when the surface temperature varies or when the virtual origin of the thermal boundary layer differs from that of the momentum boundary layer, somewhat different results will be obtained, although the effects are not large. This is a problem that can best be discussed later in comnection with a theoretical model of the thermal boundary layer. An alternative method of presentation of these results is shown on Figure 6 . Here everything is quite analogous to the very similar results for friction coefficient on Figure 3. A more convenient blowing parameter, $b_{h}=F / S t_{0}$, is introduced, and the laminar similarity solution results are superimposed. In addition, the following equation proposed by Kutateladze and Leont'ev [9], based on analytic considerations, is included.

$$
S t / S t_{0}=\left(1-b_{h} / 4\right)^{2}
$$

Figure 6 illustrates very graphically how blowing decreases the heat transfer coefficient, and this, of course, is the primary reason for the interest in transpiration as a method of protecting surfaces against high temperature fluids.

The same comments regarding blow-off as were made in connection with the friction coefficients apply here. It is, however, of interest to note that the laminar similarity solution for heat transfer does not really indicate 
a blow-off point, because for $\operatorname{Pr}=.70$ the Stanton number is still greater than zero at the point where the friction coefficient has reached zero, The Kutateladze and Leont'ev solution predicts blow-off at $b_{h}=4.0$, but over most of the range it is very close to the laminar solution. The experiments of Moffat, and of Whitten, include extensive amounts of data for $b_{h}$ equal to 4 and greater for which St is measurably above zero. It thus appears that the Kutateladze and Leont'ev solution predicts blow-off a little early.

It has been noted previously that both the friction coefficient and the Stanton number tend to be rather strong functions of local conditions along the surface rather than the history of the boundary layer up to the point in question. In other words, it has been stated that stanton number tends to be primarily a function of local enthalpy thickness Reynolds number, $\mathbb{R e}_{\mathrm{H}}$, and $F$, and is relatively independent of how $F$ has varied up to the point in question. Figures 7 and 8 , which show some of the experimental data of Whitten et al [13] illustrate this point very we11. Both a step down and a step up in $F$ are shown. Although there is in both cases a detectable recovery region, most of the adjustment to the new boundary condition occurs very rapidly. These figures represent extreme cases, so it is not surprising that when there are continuous variations of $F$ the recovery is virtually undetectable.

The friction coefficient behavior is substantially the same, although it is much more difficult to accurately measure friction coefficients just following a step in blowing.

The experimental facts that $\mathrm{C}_{f} / 2$ is primarily a function only of $\mathrm{Re}_{\mathrm{M}}$ and $\mathrm{F}$, and $\mathrm{St}$ is a function primarily only of $\operatorname{Re}_{\mathrm{H}}$ and $F$, lead to 
a relatively simple approximate procedure for calculating $\mathrm{C}_{f}$ and St along a surface where $F$ varies in some arbitrary manner. One need only suba stitute Eq. (4) into Eq. (3) and Eq. (8) into Eq. (7), and one has simple differential equations for $\mathrm{Re}_{\mathrm{M}}$ and $\mathrm{Re}_{\mathrm{H}}$ which may be integrated, at least numerically, to yield momentum and enthalpy thickness Reynolds numbers as functions of $x$. Then Eqs. (4) and (8) are used to determine $C_{f} / 2$ and St at each local point. This procedure works remarkably well, although it is, of course, restricted to the constant property boundary layer with constant free-stream velocity, and in the case of the energy equation, we have so far restricted consideration to the constant surface temperature. problem.

Whitten [13] also presents data for step changes in surface temperature with transpiration, and includes step changes in transpiration rates. With these results it is possible to use the method of superposition to build up solutions to the energy equation for cases where both $F$ and surface temperam ture vary in any arbitrary manner along the surface, and indeed Whitten has done just this [14]. However, at some point one must question whether it is not more fruitful to attempt to devise a more fundamental model of the turbulent transport processes so that all manner of variations in boundary conditions, and including varying fluid properties and viscous dissipation, can be handled with a single consistent theory based on the differential equations of the boundary layer rather than the integral equations. The constant property, constant free-stream velocity problem with arbitraxily varying $F$ and arbitrarily varying $t_{0}$, probably represents about the limit of integral methods, at least where reasonable precision is desired, although 
integral methods can be pushed considerably farther where low precision is adequate (see for example, Kays [15]). 
The Case of Varying Free-Stream Velocity - The momentum and energy integral equations, for constant properties and constant surface temperature, but now including a variable free-stream velocity, may be written in the following form:

$$
\begin{aligned}
& \frac{d \operatorname{Re}_{M}}{d \operatorname{Re}_{X}}=C_{f} / 2+F-K(1+H) \operatorname{Re}_{M} \\
& \frac{d e_{H}}{d e_{X}}=S t+F \\
& \text { where } \quad d e_{x}=U_{\infty} d x / \nu ; K=\left(\nu / U_{\infty}^{2}\right)\left(d_{\infty} / d x\right) \\
& \quad d e_{M}=d\left(U_{\infty} \delta_{2}\right) / \nu ; d \operatorname{Re}_{H}=d\left(U_{\infty} \Delta_{2}\right) / \nu
\end{aligned}
$$

One thing that is immediately apparent from these equations is that the pressure gradient, which is incorporated implicitly in the acceleration parameter $\mathrm{K}$, has a direct influence on the development of the momentum equation, but any influence on the energy equation must be an indirect one coming in through the turbulent transport properties and the velocity profile. An important simplifying feature of the previously discussed constant freestream velocity, constant surface temperature problem is that the momentum and thermal boundary layers tend to grow together, and this is one reason why relations such as Eq. (8) hold up remarkably well in the face of arbitrarily varying $F$ along the surface. It should be apparent from Eqs. (10) and (11) that such will not be the case when free-stream velocity varies. Before examining the kind of heat transfer behavior that is obtained 
when free-stream velocity varies, it is worthwhile to look at some more of the implications of Eqs. (10) and (II). A particularly interesting case arises when the acceleration parameter $\mathrm{K}$ is a positive number and constant. This is a case of an accelerated flow, and in fact, it is the accelerated flow that is obtained when the fluid is confined between two straight but converging planes. The possibility exists, according to Eq. (10), that an equilibrium will be reached such that $\operatorname{Re}_{M}$ remains constant, and indeed such flows are observed experimentally. There are obvious experimental advantages in being able to hold such an important parameter as $\mathrm{Re}_{\mathrm{M}}$ constant, and so the constant-K boundary layer has been extensively studied. Most of the experimental data obtained by the author and his colleagues for varying free-stream velocity have been for constant-K accelerating boundary layers. According to Eq. (11), however, the thermal boundary layer will continue to grow even though the momentum boundary layer has reached the equilibrium, or asymptotic condition.

It has been cbserved experimentally that strong acceleration, $1 . e$. large $\mathrm{K}$, will lead to a "retransition" of the turbulent boundary layer to a laminar boundary layer. One might predict that this would occur simply from examination of Eq. (10) alone. A large positive value of $\mathrm{K}$ will cause $\operatorname{Re}_{M}$ to decrease, and if $\mathrm{K}$ is sufficiently large $\operatorname{Re}_{M}$ will be pushed down into the region of stable laminar boundary layers. This is not to say the $R e_{M}$ is necessarily the sole criterion for "retransition", but it certainly must be an important one. Assuming for the moment that some critical value of $\operatorname{Re}_{\mathrm{M}}$ (say, 200-400) is the dominant criterion, Eq. (10) immediately suggests that positive values of $F$ will tend to 
inhibit "retransition", while negative values will aid it. This is actually what is experimentally observed. For $F=0$ "retransition" or "Iaminarization" will occur whenever $\mathrm{K}$ exceeds about $3 \times 10^{-6}$ for an extended distance along a surface. For $F$ positive (blowing) a turbulent boundary layer can be maintained at considerably higher values of $\mathrm{K}$ without "laminarization". For F negative (suction), both "laminarization" and the asymptotic suction layer are approached at values of $K$ lower than $3 \times 10^{-6}$, and it is very difficult to tell which comes first when examining experimental data.

It is experimentally observed for the case of no transpiration, $F=0$, that laminarization causes a very substantial reduction in stanton number, but that this phenomena is a continuous function of $\mathrm{K}$ rather than an abrupt change at some critical value of $\mathrm{K}$. In other words, at any positive value of $\mathrm{K}$ there will be some reduction in Stanton number (relative to what the Stanton number would have been at the same value of $\operatorname{Re}_{H}$ for $K=0$ ). On the basis of these facts, one might be led to conclude that blowing, under accelerating free-stream conditions, would lessen the decreasing Stanton number tendency, while suction would enhance it.

On Fig. 9, the results of two test-runs reported by Thielbahr,et a1 [16] are plotted. In the upper part of the diagram, there is a case of mild blowing, $F=0.001$, starting with constant free-stream velocity, followed by a relatively strong acceleration, $K=1.47 \times 10^{-6}$, and finally reverting to constant free-stream velocity. The acceleration does, indeed, appear to cause a small reduction in Stanton number. At the end of the acceleration, there appears to be a start of a recovery back toward the line for $k=0$, but it is only a partial recovery. A reason for the failure of recovery would be found if one examined the values of $R e_{M}$ and $\operatorname{Re}_{H}$ in the recovery region. 
$\mathrm{Re}_{\mathrm{H}}$ is very much greater than $\mathrm{Re}_{\mathrm{M}}$, and $\mathrm{Re}_{\mathrm{M}}$ catches up only very slowly, a fact that an examination of Eqs. (10) and (11) would make apparent.

The second set of data on Fig. 9 shows a case of a rather strongly blown boundary layer, $F=0.006$, that develops at constant free-stream velocity, and then is subjected to a relatively mild acceleration, $\mathrm{K}=0.8 \times 10^{-6}$. Surprisingly, the effect of acceleration is precisely the reverse of the previous case. Stanton number tends to increase above the value for no acceleration.

Attention has been drawn to these examples merely to illustrate the fact that the combination of transpiration and varying free-stream velocity results in complex interactions that are not going to be understood unless the basic turbulent transport mechanisms are studied in more detail. It does not appear practicable or fruitful to attempt to describe the kind of behavior shown on Fig. 9 by use of the integral equations and integral correlations. Let us examine a more fundamental approach. 
Some Models for Solution of the Momentum Differential Equation of the Boundary Layer - The digital computer has, in a period of no more than five or six years, revolutionized turbulent boundary layer theory and turbulent boundary layer prediction methods. Stable, accurate finite-difference solutions to the momentum, energy, and mass diffusion equations of the boundary layer have become practicable. Fast, economic procedures are available which include the possibility of an infinite variety of boundary conditions as well as the inclusion of variable fluid properties, viscous dissipation, chemical reaction, etc. It is not the present intention to extoll the virtues of any particular procedure; actually there are several very good ones in common use. However, the accuracy of any of the calculation procedures depends upon the basic physics introduced into it, and this is our present concern.

The time-average momentum equation of the boundary layer, particularized for the moment to constant fluid properties, and neglecting nomal turbulent stresses, may be written as follows:

$$
\bar{u} \frac{\partial \bar{u}}{\partial x}+\bar{v} \frac{\partial \bar{u}}{\partial y}-\frac{\partial}{\partial y}\left[v \frac{\partial \bar{u}}{\partial y}-\overline{u^{\prime} v^{\prime}}\right]+\frac{g_{c}}{\rho} \frac{d \bar{p}}{d x}=0
$$

If the turbulent shear stress $\overline{u^{1} v^{7}}$ were known at all points in the boundary layer, the momentum problem simply becomes one of solution of $\mathbb{E}$. (12) for any desired boundary conditions, including transpiration. As dism cussed above, numerous adequate finite-difference methods are available to do this. 
Although progress continues to be made in turbulent transport theory in genera1, and turbulent boundary layer theory in particular, it is stil1 fair to say that there is as yet no truly fundamental turbulence theory that may be used as a universal starting point for solution of turbulence problems. Turbulent boundary layer theory has gone through, and continues to go through, a series of etages involving successively higher orders of sophistication. Each step in this process involves the correlation of experimental data at a more fundamental level, opening up the possibility of solving successively broader ranges of problems with a single consistent set of empirical constants. The information and calculating procedures to be presented here do not represent any very bold steps toward a more general theory. Higher order models are presently being investigated by numerous researchers, and hopefully will lead to theories that embrace still broader classes of applications, although probably at the price of complexity and computation cost. In the meantime, it will be demonstrated here that a relatively simple model can be used to adequately predict the behavior of the transpired boundary layer over a sufficiently wide range of conditions to make it a very practicable tool for engineering design。

We will first introduce the concept of eddy diffusivity for momentum, $\varepsilon_{N_{1}}$, as a convenient way of expressing the turbulent shear stress.

$$
\overline{u^{\prime} v^{\prime}}=-\xi_{M} \frac{\partial \bar{u}}{\partial y}
$$

Already, we are in the realm of theoretical controversy, but the eddy diffusivity concept has the computational virtue of allowing one to use the 
same computation program for both laminar and turbulent boundary layer. Since most turbulent boundary layers grow out of laminar boundary layers, the adm vantage is obvious.

It is convenient to visualize the turbulent boundary layer as consisting of an inner wall-dominated region, and an outer region which physically occupies most of the thickness of the boundary layer. However, for noet applications the inner region turns out to be by far the more important one, and it is to this region that we will now focus primary attention.

The inner region is characterized by a region immediately adjacent to the wall in which viscous forces predominate $\left(\overline{u^{1} v^{7}}\right.$ approaches zero), and a region farther out in which momentum transfer is almost entirely by turbulent transport processes, but in which the scale and intensity of the turbulence is stil1 strongly dependent upon the proximity of the wall. Between these is a transition region in which both mechanisms are important in varying degrees。 Some analysts prefer to represent the entire inner region by an empirically established "law-of-the-walI", but probably the most significant conclusion that has come out of the experimental work upon which this paper is based is that the thickness of the viscous-dominated part of the inner region is strongly dependent upon both transpiration and free-stream pressure gradient, and thus no single law-of-the-wall can be adequate.

The Prandt 1 mixing-length theory, despite much criticism for many years, still provides a remarkably adequate basis for describing the turbulent momentum transport process in the inner region. The mixing-length, $l$, is defined such that it is related to the eddy diffusivity for momentun and the mean velocity gradient by the following equation: 


$$
\varepsilon_{\mathrm{M}}=\ell^{2} \frac{\partial \overline{\mathrm{u}}}{\partial \mathrm{y}}
$$

Outside of the viscous-dominated region immediately adjacent to the wal1, the mixing-length in the inner region of the boundary layer seems to be proportional to distance y from the wall, with a proportionality factor, $k$, which can be assumed to be independent of either transpiration rate or pressure gradient. Whether or not $k$ is a truly universal constant is not highly important, because another constant to be described shortly can be used to absorb any dependence on transpiration or pressure gradient; the experimental data are not sufficiently accurate to justify finer discrimination. Thus we will model the region outside of the viscous near-wall region. (which we will now term the viscous sublayer), but inside of the outer, or "wake", region, by:

$$
\ell=\mathrm{ky}
$$

The viscous sublayer can be modelled in a simple way by introducing a damping function that forces the mixing-length $\ell$ to zero at the wall. Designating the damping function as $D$, the mixing-length may then be expressed as:

$$
\ell=\mathrm{kyD}
$$


The damping function $D$ can be any function that is equal to zero at the wall and equal to unity at large values of $y$, but obviously some functions will model the actual experimental data better than others. The simplest possible damping function idealizes the sublayer as a finite, purely viscous region as follows:

$$
\begin{aligned}
& D=0.0 ; \quad y^{+} \leq<y_{\text {crit }}^{+} \\
& D=1.0 ; \quad y^{+}>y_{\text {crit }}^{+}
\end{aligned}
$$

$\mathrm{y}^{+}$is the non-dimensional distance from the wall expressed in so-called wall coordinates. $\mathrm{y}_{\text {crit }}^{+}$is then the effective viscous sublayer thickness. For the flat-plate case with no pressure gradient and no transpiration, $y_{c r i t}^{+}$is measured to be approximately 11.0. For accelerating flows, $y_{\text {crit }}^{+}$increases, for transpiration $y_{\text {crit }}^{+}$decreases, and for suction $\mathrm{y}_{\text {crit }}^{+}$increases. A reasonably adequate scheme can be developed using this very simple damping function, its main deficiency being that it does not model the velocity profile very well in the $\mathrm{y}^{+}$range 5 to 30 。 Another scheme which is very popular today was first suggested by Van Driest [17]. The Van Driest damping function is exponential, so that the influence of the viscous region decays smoothly from the wall and is still felt as far out $\mathrm{y}^{+}=50$. The simplest version of the Van Driest damping function is that given in the following equation:

$$
D=1.0-\exp \left(-y^{+} / A^{+}\right)
$$


In this case $\mathrm{A}^{+}$becomes an effective viscous sublayer thickness that must be established experimentally. For the flat-plate case, $A^{+}=26.0$ is approximately what is obtained. However, the precise value of $\mathrm{A}^{+}$ depends to a certain extent upon the value of the mixing-length constant $k$. Fig. 10 shows the values of $\mathrm{A}^{+}$that are obtained for differing values of $\mathrm{k}$ to yield identical values of $\mathrm{u}^{+}$at an arbitrary $\mathrm{y}^{+}=80.0$. There is some evidence that at low $\operatorname{Re}_{M}$, i.e., below $6000, \mathrm{k}$ tends to increase (see Simpson [18]), but the data upon which this paper is based are not conclusive in this regard, and $k=0.44$ is used for all of the illustrative calculations to follow. Those preferring other values of $k$ can use Fig. 10 as a basis for changing all of the functions, as will be seen later. Like $\mathrm{y}_{\text {crit }}^{+}, \mathrm{A}^{+}$is found to be a function of both a pressure gradient parameter and a transpiration parameter (and perhaps other things yet to be investigated).

The non-dimensional distance from the wall $\mathrm{y}^{+}$contains within it the wall shear stress $\tau_{0} \cdot A^{+}$is simply the value of $y^{+}$at the effective outer edge of the sublayer, and thus is also normalized with respect to $\tau_{0}$. It can be argued that in the region under consideration, a local Reynolds number of turbulence can be defined as

$$
\operatorname{Re}_{t}=l_{t} \sqrt{\mathrm{u}^{\top} v^{\top}} / \nu=l_{t} \sqrt{g_{c} \tau_{t} / \rho} / \nu
$$

$\ell_{t}$, the turbulence length scale, is effectively equal to the mixing-length $\ell=\mathrm{ky}$. Thus,

$$
\operatorname{Re}_{t}=k y \sqrt{g_{c} \tau_{t} / \rho} / \nu
$$


In the flat-plate case, the shear stress $\tau$ is essentially equal to $\tau_{0}$ out to a distance well beyond the viscous sublayer, so that $\operatorname{Re}_{t}$ is equal to $\mathrm{ky}^{+}$. Thus $\mathrm{A}^{+}$can be interpreted as a critical value of the local Reynolds number of turbulence, which determines the viscous sublayer thickness.

If this idea has ary merit, howerer, accounc should be taken of the fact that for flows with pressure gradients in the flow direction, or for transpired boundary layers, the local shear stress can vary markedly with $y$ in the region near the wall, in which case the local shear stress ought to be used in the damping function rather than the wall shear stress. This notion has led to the following alternative form for the Van Driest damping function

$$
\begin{aligned}
& D=1.0-\exp \left(-y^{+} \sqrt{\tau^{+}} / A^{+}\right) \\
& \text {where } \tau^{+}=\tau / \tau_{0}
\end{aligned}
$$

Eq. (20) is appealing because it immediately suggests that for strongly accelerating flows, where $\tau^{+}$decreases very sharply with increasing $y^{+}$, it would be possible to have a sufficiently strong pressure gradient so that D would remain a very small number throughout the boundary layer. This, of course, would correspond to the observed phenomena of laminarization. In. other words, this would be a situation where $\mathrm{Re}_{t}$ never exceeds a critical value of the Reynolds number of turbulence, $\mathrm{A}^{+}$, and the entire boundary layer remains laminar. Similarly, the thinning of the laminar sublayer that is observed for blowing is correctly modelled, as well as the opposite trend for suction. These features have led some analysts to prefer Eq. (20) over 
Eq. (18) as a basis for defining $\mathrm{A}^{+}$. It is important to recognize this difference (and there are still other schemes) because otherwise it is difficult to compare the results of different workers.

Despite the fact that Eq. (20) with $\mathrm{A}^{+}=26.0$ yields the trends that are observed experimentally, the observed effects are considerably greater. Thus for transpiration and/or pressure gradient it still becomes necessary to express $\mathrm{A}^{+}$as a function of a transpiration parameter and a pressure gradient parameter. Using wall coordinates, these are respectively $\mathrm{v}_{\mathrm{w}}^{+}$and $\mathrm{P}^{+}$(defined in the Nomenclature). All that is accomplished by using Eq. (20) in preference to Eq. (18) is to somewhat lessen the dependence of $\mathrm{A}^{+}$upon these parameters.

Values of $\mathrm{A}^{+}$as defined by Eq. (20) have been determined from a wide range of experimental velocity profiles for blowing, suction, acceleration, and combinations of acceleration and transpiration, from the work of Simpson et al [10], Julien et a1 [19], and Loyd et al [20]. The following equation is an empirical correlation of these results and fits them all with a tolerance of about \pm 10 percent.

$$
\mathrm{A}^{+}=\frac{\mathrm{A}_{\mathrm{O}}^{+}}{5.15\left[\mathrm{v}_{\mathrm{W}}^{+}+\frac{5.86 \mathrm{P}^{+}}{\left(1+5.0 \mathrm{v}_{\mathrm{W}}^{+}\right)}\right]+1}
$$

where $A_{0}^{+}$is the value of $A^{+}$for no transpiration and no pressure gradient, as taken from Fig. 10. Eq. (21) is plotted on Figure 11 for the case of $k=0.44$. Here the effects of a favorable pressure gradient, and the effects of transpiration are clearly seen. Although the equation itself extends to positive values of $\mathrm{P}^{+}$, and may well be valid there, the experimental data upon which $\mathrm{Eq} .(21)$ is based are al1 for the case of accelerating flows (negative $\mathrm{P}^{+}$). 
Another damping function, proposed by Evans et al [21], utilizes a linear function as follows:

$$
\begin{aligned}
D & =\mathrm{y}^{+} / \mathrm{B}^{+} ; \mathrm{y}^{+} \leq \mathrm{B}^{+} \\
& =1.00 ; \mathrm{y}^{+}>\mathrm{B}^{+}
\end{aligned}
$$

In this case $\mathrm{B}^{+}$becomes an effective viscous sublayer thickness. This function involves a discontinuity, but only in $D$, not in either mixing length or the resulting velocity profile. For very high Prandtl number fluids, the heat transfer behavior turns out to be very sensitive to the behavior of the damping function at small values of $\mathrm{y}^{+}$. It is important to note that the van Driest function is also linear at small values of $\mathrm{y}^{+}$, so either scheme seems equally attractive from this point of view. (Both schemes result in $\varepsilon_{M}$ varying as $\mathrm{y}^{+^{4}}$ as the wall is approached). The Evans damping function seems to give velocity profiles that are a little closer to the experimental data at values of $\mathrm{y}^{+}<100$, and therefore is especially attractive for low Reynolis number work.

Like $\mathrm{A}^{+}$, the value of $\mathrm{B}^{+}$depends to a certain extent upon the value of mixing-length constant $k$ used. Fig. 10 shows this dependence.

Values of $\mathrm{B}^{+}$have been determined from the experimental data cited above, and these results are correlated within about \pm 10 percent by the following equation.

$$
\mathrm{B}^{+}=\frac{\mathrm{B}_{\mathrm{O}}^{+}}{\left[9.0 \mathrm{v}_{\mathrm{w}}^{+}+\frac{3.35 \mathrm{P}^{+}}{1.0+4.0 \mathrm{v}_{\mathrm{w}}^{+}}\right]+1}
$$


Again, $\mathrm{B}_{0}^{+}$is taken from Figure 10. Eq. (23) is plotted on Fig. 12 for the case of $k=0.44$. The behavior is very similar to that of $A^{+}$; there is a somewhat stronger effect of $\mathrm{v}_{\mathrm{w}}^{+}$and $\mathrm{P}^{+}$, but this is due to the fact that Eq. (20) contains the shear stress ratio $\tau^{+}$. Had the comparison. been made with $\mathrm{A}^{+}$defined as in $\mathrm{Eq} \cdot(18)$, it would be found that $\mathrm{B}^{+}$differs from $\mathrm{A}^{+}$by almost a constant factor.

Figs. 13 and 14 show examples of the inner region velocity profiles (i.e., the law-of-the-wa11) as predicted by integration of Eq. (12) using the $\mathrm{B}^{+}$scheme, and a comparison with experimental data, in the $\mathrm{y}^{+}$range 10 to 100 (the temperature profile on Fig. 13 will be discussed presently.) The data on Fig. 13 are from several test runs for $\mathrm{v}_{\mathrm{w}}^{+}=0.0$ and $\mathrm{p}^{+}=0.0$; they correspond closely to the data that are generally found in the literature for this basic case. Fig. 14 shows two cases of blowing alone, and one case of a combination of blowing and acceleration. As can be seen, the prediction is excellent; the $\mathrm{A}^{+}$scheme is also quite adequate, but slightly underpredicts $\mathrm{u}^{+}$in the $\mathrm{y}^{+}$range $10-50$ 。

A word should now be said about the reasons for the observed dependence of $\mathrm{A}^{+}$or $\mathrm{B}^{+}$upon $\mathrm{P}^{+}$and $\mathrm{v}_{\mathrm{W}}^{+}$. It was suggested earlier that the outer edge of the viscous sublayer might be characterized by some critical value of the local Reynolds number of turbulence, Eq. (19), and indeed this hypothesis does yield the correct trends with $\mathrm{v}_{\mathrm{w}}^{+}$and $\mathrm{P}^{+}$. This scheme applied to $\mathrm{A}^{+}$yields somewhat better results than if applied to $\mathrm{Y}^{+} \mathrm{crit}^{+}$. If applied to $\mathrm{B}^{+}$, it works better still. Since each of these three schemes employs an effective sublayer thickness that is greater than the previous one, it appears possible that the value of $\mathrm{y}^{+}$at which fully developed self-sustaining turbulence obtains, and therefore at which the Reynolds number of 
turbulence has exceeded some critical value, is at a value of $\mathrm{y}^{+}$greater than the effective sublayer thickness used in these models. An investigation by Andersen [22] based on the experimental data cited above, has revealed the interesting fact that if $\mathrm{y}^{+}$equal to about 2 times $\mathrm{B}^{+}$is choser as the critical point, the local Reynolds number of turbulence is very close to the same number for all of the data examined. These data include some 37 test runs covering a wide range of transpiration, acceleration, and combinations of transpiration and acceleration. To be more precise, at $\mathrm{y}^{+}=2 \mathrm{~B}^{+}$, the Reynolds number of turbulence, $\operatorname{Re}_{t}$, is equal to about 33.0 over the entire range of tests. In other words, with this fact alone it is possible to calculate $\mathrm{B}^{+}$as a function of $\mathrm{P}^{+}$and $\mathrm{v}_{\mathrm{W}}^{+}$and to obtain results that correspond remarkably closely to those represented by Eq. (23), or Fig. 12. Thus the concept that the thickness of the viscous sublayer is determined by a critical Reynolds number of turbulence seems at the present time to be an attractive one.

$\mathrm{A}^{+}$and $\mathrm{B}^{+}$as represented by Eqs. (21) and (23), were obtained under what might best be described as equilibrium conditions, i.e., conditions under which $\mathrm{v}_{\mathrm{W}}^{+}$and/or $\mathrm{P}^{+}$are invariant or at worst are varying only slowly along the surface. Under non-equilibrium conditions where these parameters are changing rapidly or abruptly, it has been observed that the sublayer does not change abruptly to its new equilibrium condition, $i, e, B^{+}$does not immediately assume its new equilibrium value. Given the stability nature of the problem, this is probably not surprising. It can be hoped that some of the higher order models of turbulence will predict this effect, but in the meantime, a reasonably satisfactory expedient is to use a rate equation of a type suggested by Launder [23]: 


$$
\begin{gathered}
\frac{\mathrm{dB}_{\mathrm{eff}}^{+}}{\mathrm{dx}^{+}}=\left(\mathrm{B}_{\mathrm{eff}}^{+}-\mathrm{B}_{\mathrm{eq}}^{+}\right) / \mathrm{C} \\
\quad\left(\mathrm{or} \mathrm{A}_{\mathrm{eff}}^{+} \text {and } \mathrm{A}_{\mathrm{eq}}^{+}\right)
\end{gathered}
$$

$\mathrm{B}_{\text {eff }}^{+}$is the effective value of $\mathrm{B}^{+}$, while $\mathrm{B}_{\text {eq }}^{+}$is the equilibrium value obtained from Eq. (23). A value of $\mathrm{C}$ of about 4.0 has been found to he reasonable。

AII of the discussion up to now has been concerned with the inner region of the boundary layer. The outer region, comprising the greater part of the boundary layer thickness, is of considerably less importance in predicting performance, and thus can be handled successfully using more gross approximations. This statement may not be valid for very non-equilibrium boundary layers under adverse pressure gradient conditions, but how valid it actually is for accelerating flows and for transpired boundary layers with and without acceleration, will be demonstrated 1ater. In any case, for equilibrium or near equilibrium boundary layers, either the assumption of a constant value of eddy diffusivity over the entire outer region, or the assumption of a constant value of mixing-length over the entire outer region yields approximately the same result. If constant eddy diffusivity is used, an empirical correlation of eddy diffusivity as a function of momentum thickness Reynolds number can be obtained. However, if mixing-length is used in the inner regions, it is probably computationally simpler to use the mixing-length concept for the entire boundary layer. A satisfactory scheme is to express the outer region mixing-length as a fraction, $\lambda$ of the total boundary layer thickness. 
$\lambda=0.085$ or 0.080 , based on the $99 \%$ boundary layer thickness, works remarkably well for the entire range of test data on which this paper is based. One simply evaluates $\&$ from Eq. (15) until the value obtained exceeds $\ell=\lambda \delta .99$, and then uses the latter value for the remainder of the boundary layer.

At values of ' $e_{\text {IM }}$ less than about 6.000 , there is some evidence that $\lambda$ is greater. The following is an empirical fit that works reasonably satisfactorily。

$$
\lambda=0.25 \operatorname{Re}_{M}^{-1 / 8} ; \operatorname{Re}_{M} \leq 6000
$$

Boundary layer predictions for strong blowing are somewhat improved if this latter correction is not made. This may actually be indirect evidence that the mixing-length constant, $k$, is lowered by strong blowing, but this kind of discrimination is very difficult to make from the available data. 
A Model for Solution of the Energy Differential Equation of the Boundary

Layer - The time-averaged energy equation of the boundary layer, particularized to constant fluid properties and negligible viscous dissipation, and neglecting turbulent conduction in the stream direction, may be written as follows:

$$
\bar{u} \frac{\partial \bar{t}}{\partial x}+\bar{v} \frac{\partial \bar{t}}{\partial y}-\frac{\partial}{\partial y}\left[\alpha \frac{\partial \bar{t}}{\partial y}-\overline{t^{\prime} v^{\prime}}\right]=0
$$

This equation can be solved for any desired boundary conditions providing that the velocity field has been established first by solution of the momentum equation, and providing that we have information on $\overline{t^{8} v^{8}}$. For convenience we introduce the concept of eddy diffusivity for heat, $\varepsilon_{\text {I }}$ "

$$
\overline{t^{\prime} v^{\gamma}}=-\varepsilon_{H} \frac{\partial \bar{t}}{\partial y}
$$

Although it might be fruitful to attempt to evaluate $\overline{t^{1} v^{7}}$ or $\varepsilon_{H}$ on the basis of assumptions that are independent of the turbulent shear stress, it seems apparent that there is some kind of relationship between $\overline{t^{\prime} v^{\top}}$ and $\overline{u^{\prime} v^{\top}}$, or $\varepsilon_{\mathrm{H}}$ and $\varepsilon_{\mathrm{M}}$. Therefore most analysts have found it convenient to introduce the concept of turbulent Prandtl number, $\mathrm{Pr}_{\mathrm{t}}$, defined as follows:

$$
\operatorname{Pr}_{t}=\frac{\xi_{M}}{\xi_{H}}
$$

Introducing Eqs. (27) and (28) into Eq. (26) we obtain: 


$$
\bar{u} \frac{\partial \bar{t}}{\partial x}+\bar{v} \frac{\partial \bar{t}}{\partial y}-\frac{\partial}{\partial y}\left[\left(\alpha+\varepsilon_{M} / \operatorname{Pr}_{t}\right) \frac{\partial \bar{t}}{\partial y}\right]=0
$$

If $\mathrm{Pr}_{t}$ were known, Eq. (29) could be solved for any desired boundary conditions so long as the momentum equation must be solved anyway. Evaluation of the turbulent Prandt number is then one of the central problems of turbulent heat transfer.

A very simple physical model of the turbulent momentum and energy transfer process leads to the conclusion that $\xi_{H}=\xi_{M}$, i.e., $\operatorname{Pr}_{t}=1.00$ (the "Reynolds Analogy"). Slightly more sophisticated models suggest that $\operatorname{Pr}_{t}>1.00$ for $\operatorname{Pr}<1.00$, and still other models suggest that $\operatorname{Pr}_{t}$ equals 0.7 or 0.5 in turbulent wakes. The experimental data are not abundant, but Figs. 15 and 16 show the measurements, respectively, of Simpson, Whitten, and Moffat [24], and of Kearney, Moffat, and Kays [25], with air as a working substance. These were all evaluated from measurements of the slopes of mean velocity and temperature profiles, together with estimates of shear stress and heat flux distributions, and the experimental uncertainty is high. The data on Fig. 15 are all for constant free-stream velocity, but cover a wide range of blowing and suction conditions. The data on Fig. 16 are for accelerated boundary layers with a considerable range of blowing and suction. The choice of $\mathrm{y}^{+}$as a basis for comparison is made only because a slightly better correlation is obtained than with other parameters, but even this fact is debatable. However, despite the very considerable scatter of data, two conclusions seem definitely warranted. First, the turbulent Prandt1 number, at least for air, has an order of magnitude of unity. This fact alone is significant, for it provides considerable justification for 
use of the concept of eddy diffusivity in the first place. The second conclusion is that $\operatorname{Pr}_{t}$ is evidently less than 1.00 in the wake or outer region, while it is greater than 1.00 near the wall. Very near the waII the experimental uncertainty becomes excessive, so that it is not possible to establish any limiting value in this rather critical region.

Another way of evaluating turbulent Prandt1 number from experimental temperature profiles is to determine by computer experiments the turbulent Prandt1 number function that must be used in order to predict given experimental temperature profiles using Eq. (29) and any one of the models for $\xi_{M}$ previously discussed. In effect the experimental velocity profile Is partially removed from consideration, but different results for $\operatorname{Pr}_{t}$ will be obtained depending upon how well the model for $\varepsilon_{M}$ reproduces true velocity profiles.

Using this scheme, the results on Fig. 17 for the inner region were obtained using the $\mathrm{B}^{+}$damping function, Eq. (22), and experimental data for several cases of blowing and acceleration, as well as some data based on the average of several non-transpired, non-accelerated runs. $B^{+}$for each run. was evaluated from the corresponding velocity profile by a computer experiment that forced the computed and measured velocity profiles to match at an arbitrary $\mathrm{y}^{+}=80$. The results are thus independent of velocity measurements at very small values of $\mathrm{y}^{+}$. For $\mathrm{y}^{+}>30$ there seems no question that the most probable value of $\operatorname{Pr}_{t}$ is simply 1.00 , i.e., the Reynolds Analogy, regardless of transpiration or acceleration. On the other hand there is a definite indication of much higher $\operatorname{Pr}_{t}$ for lower $\mathrm{y}^{+}$. Note of course that since only $\mathrm{y}^{+}<100$ is being considered, the lower $\mathrm{Pr}_{t}$ 
that seems characteristic of the outer region is not seen.

The same computer experiment performed with the $\mathrm{A}^{+}$damping function tends to yield values of $\operatorname{Pr}_{t}$ that average about 0.9 for $y^{+}>30$, but otherwise the same conclusions are reached. However, since the $\mathrm{B}^{+}$scheme generally predicts the velocity profiles more accurately in the region $y^{+}<100$, it would seem that the results on Fig. 17 more nearly represent the truth. (The term "truth" here must be qualified, because if $\mathrm{A}^{+}$is being used in a boundary layer prediction scheme, it is necessary to use values of $\operatorname{Pr}_{t}$ based on $\mathrm{A}^{+}$, even though these values differ from what might be measured directly.) The absolute necessity for a high $\operatorname{Pr}_{t}$ at very low values of $y^{+}$ is well illustrated by the data and calculations for temperature profile shown on Fig. 13 for the case of no transpiration and no pressure gradient. For $\mathrm{y}^{+}$greater than about 30 two parallel lines describe the velocity and temperature profiles very well. This can be interpreted as meaning that $\operatorname{Pr}_{t}=1.00$. However, if an attempt is made to predict the temperature profile with $\mathrm{Pr}_{t}=1.00$ throughout, the result is as shown. A higher $\mathrm{Pr}_{\mathrm{t}}$ is needed for $y^{+}<30$ to avoid badly underpredicting $t^{+}$in the $y^{+}$range 30 to 100. Closer examination reveals that it makes little difference how high $\operatorname{Pr}_{t}$ is for $\mathrm{y}^{+}<10$, and at $\mathrm{y}^{+}=15$ it is already too late to introduce the correction. Apparently for $\mathrm{y}^{+}<15$ turbulent velocity fluctuations result in a considerably greater rate of transport of momentum than of heat for reasons that are not yet fully understood. The heat conduction model of Jenkins [25] would predict this trend, but not the concentration of the effect in this narrow region.

At the present time an empirical correlation of this effect seems the 
most practicable expedient if heat transfer rates are to be calculated. The assumption of a constant value of $\operatorname{Pr}_{t}=0.9$ throughout the boundary layer will yield overall heat transfer rates that are quite satisfactory: temperature profiles can be more accurately predicted if a variation of $\mathrm{Pr}_{t}$ with $\mathrm{y}^{+}$is introduced. The following correlations have been used successfully by the writer for air:

If $\mathrm{A}^{+}$is used as the damping function (Eq. 20):

$$
\begin{array}{r}
\operatorname{Pr}_{t}=\left(1.43-0.17 \mathrm{y}^{+1 / 4}\right)\left(1+\mathrm{v}_{\mathrm{w}}^{+}\right) \\
\text {If } \operatorname{Pr}_{t}<0.85 ; \operatorname{Pr}_{t}=0.85
\end{array}
$$

The dependence upon $\mathrm{v}_{\mathrm{W}}^{+}$in Eq. (30) is not based on the raw $\mathrm{Pr}_{t}$ data in Figs. 15 and 16, but rather upon computer experiments using the $A^{+}$scheme for evaluation of $\varepsilon_{M}$.

If $\mathrm{B}^{+}$is used as the damping function:

$$
\begin{aligned}
& \operatorname{Pr}_{t}=1+.35\left[1+\cos \left(\pi y^{+} / 37\right)\right] ; y^{+}<37 \\
& \operatorname{Pr}_{t}=1.00 ; y^{+}>37 \\
& \operatorname{Pr}_{t}=0.60 ; y>\left(\lambda \delta_{.99} / \mathrm{k}\right)
\end{aligned}
$$

Figure 13 shows an example of an inner region temperature profile prediction using the $\mathrm{B}^{+}$damping function, and Eq. (31). Actually there are any number of $\operatorname{Pr}_{t}$ functions that work equally well, and the cosine function in Eq. (31) has no physical significance. 
Some Examples of Predictions of Difficult Cases - To illustrate the quality of turbulent boundary layer predictions which can be made with a finite difference program using some of the material presented in the preceding sections, two rather difficult cases have been chosen. A modification of the Spalding/Patankar [27] program was used, although any good finite difference should yield similar results.

The first of these is illustrated on Fig. 18. Here is a case of rather strong blowing, $F=0.0058$, subjected to a moderately strongly accelerated free-stream along about half the length of the test section, with constant free-stream velocity thereafter. The variation of $U_{\infty}$ is shown at the top of the diagram. This figure then shows a comparison between measured and predicted values of $R e_{M}, C_{f} / 2$, and $S t$, plotted as functions of distance $\mathrm{x}$ along the test surface. (The fluid used was room temperature air with small temperature differences, as was the case for all of the data considered in this paper). Note that $\operatorname{Re}_{\mathrm{M}}$ is almost constant throughout the accelerated region, a consequence of a constant-K accelerated boundary layer, as discussed earlier. Following acceleration $\mathrm{Re}_{\mathrm{M}}$ then increases rapidly. The comparison between experiment and prediction must. be considered excellent, especialiy when experimental uncertainty is taken into consideration. This prediction was made using the $\mathrm{A}^{+}$damping function, but the $\mathrm{B}^{+}$scheme will do equa11y as well. Figures 19 and 20 are in some ways more impressive, for they show two velocity profiles and two temperature profiles from the same test run, in real dimensional coordinates, and a comparison between experiment and predictions. In each case the profile at $x=45.6$ inches is one taken in the 
accelerated region, while the other profile at $x=69.7$ inches is taken in the recovery region farther downstream.

Another difficult case is shown on Fig. 21. In this run, the Elow starts at constant free-stream velocity but with moderately strong blowing, $F=.004$. This flow is then subjected to a very strong acceleration starting at $x=2$ feet. In approximately the middle of the accelerated region the blowing is removed entirely. Then at about $x=3.4$ feet the acceleration is removed, and for the remainder of the test section there is no blowing and no acceleration. The Stanton number prediction shown here was obtained using the $\mathrm{A}^{+}$scheme, although the outer region of the boundary layer was calculated using a higher order turbulence model than the simple mixing-length model described here. The turbulent kinetic energy equation was solved, and $\varepsilon_{M}$ was determined from an assumed relationship between $\varepsilon_{M}$ and the kinetic energy of turbulence. However, it has been found that in flows of this type, use of a turbulent kinetic energy model in the outer part of the boundary layer contributes very little, and to all intents and purposes identical results will be obtained with the simple mixing-length model。

The important thing to note here is that the model responds remarkably to the abrupt changes in boundary conditions, and predicts the resulting non-equilibrium boundary layer very well indeed. Of particular significance is the abrupt rise in Stanton number following the removal of blowing. The ability of the prediction to follow the data at this point is heavily dependent upon the use of the rate equation and lag constant, Eq. (24). 
Future Work - The needed future research on heat transfer to the transpired turbulent boundary layer can be subdivided into two general categories. The first of these is research that is concerned with the turbulent boundary layer in general, while the second is research particularized to the transpired case. All advances in the general category will contribute to a better understanding of the transpired boundary layer. Mention has already been made of investigations into higher order models of turbulence, and as time goes on the results of these investigations can be expected to find their way into transpired turbulent boundary layer theory and prediction methods. The problem of turbulent Prandt number, or more generally, the problem of the turbulent transport of thermal energy, should provide a particularly fruitful area for research in the near future. This is obviously not a new problem; it was of great interest two decades ago when liquid metal heat transfer was first being actively investigated. However, it is clear that a better theory is needed than is presently available.

For the particular case of the the transpired turbulent boundary layer, the first obvious problem for which there is presently inadequate experimental data is the case of an adverse pressure gradient. Actually, the case of blowing and adverse pressure gradient is going to lead to early stall, so it is probably the suction problem with an adverse pressure gradient that provides the most scope for experimental work. It should be noted that Eqs. (21) and (23) will yield some numbers for use with adverse pressure gradients, and since these numbers can be predicted by a theory that also predicts the other cases relatively satisfactorily, one might be temped to use these equations for predicting the adverse pressure gradient cases. 
However it should be emphasized that no experimental data for the adverse pressure gradient cases has been used to generate these empirical equations. In attempting to apply the transpiration results and theories presented, two obvious practical problems arise. Real transpiration surfaces frequently are aerodynamically rough, and real transpiration surfaces frequently are constructed by drilling a large number of small discrete holes in a surface rather than constructing the surface from a sintered powder as was done for all of the experimental data considered here. The roughness problem is presumably not totally unlike the roughness problem for the non-transpired case, although the effects of transpiration are at the present time completely unknown. The large hole problem opens up an entire field of investigation because the number of possible geometrical variables increases enormously. There is an entire spectrum of problems lying between what might be called pure transpiration, with surface holes and spacing small relative to the laminar sublayer thickness at one end, and film cooling at the other end.

In a sense, the present paper has been concerned with only an idealized case lying at one end of a very broad spectrum of turbulent boundary layer problems. 
References

1. Rubesin, M. W., "An Analytical Estimation of the Effect of Transpiration Cooling on the Heat Transfer and Skin Friction Characteristics of a Compressible Turbulent Boundary Layer", NACA TN 3341, (1954)。

2. Dorrance, W. H., Dore, F. J., "The Effect of Mass Transfer on the Compressible Turbulent Boundary Layer Skin Friction and Heat Transfer", J.A.S.g Vol. 21, p. 404, (1954).

3. Mickley, H. A., Ross, R. C., Squyers, A. L., Stewart, W. E。, "Heat, Mass, and Momentum Transfer for Flow Over a Flat Plate with Blowing or Suction", NACA TN 3208, (1954).

4. Mickley, H. S. and Davis, R. S., "Momentum Transfer for Flow Over a Flat Plate with Blowing", NACA TN 4017, (1957).

5. Stevenson, T. N., "A Law of the Wall for Turbulent Boundary Layers with Suction or Injection", Cranfield College of Aero. Report 166, (1963).

6. McQuaid, J., "Incompressible Turbulent Boundary Layers with Distributed Injection", Ph.D. Thesis, Cambridge University, (1966).

7. Black, T. J. and Sarnecki, A。J॰, "The Turbulent Boundary Layer with Suction or Injection", A.R.C.RM 3387, (1965).

8. Romanenko, P. No and Karchenko, V. No, "The Effect of Transverse Mass Flow on Heat Transfer and Friction Drag in a Turbulent Flow of a Compressible Gas Along an Arbitrary Shaped Surface", Int. Jn. of Heat and Mass Transfer, Vo1. 6, No. 8 (1963)。

9. Kutateladze, S. S., Leont'ev, Turbulent Boundary Layers in Compressible Gases, Academic Press (1964), pp。67-86.

10. Simpson, R. L., Moffat, R. J., and Kays, W. M., "The Turbulent Boundary Layer on a Porous Plate: Experimental Skin Friction with Variable Injection and Suction", Int. Jn. Heat and Mass Transfer, Vo1. 12, pp.771-789, (1969).

11. Squire, L. C., "The Constant Property Turbulent Boundary Layer with Inm jection; A Reanalysis of Some Experimental Results", Int. In. Heat and Mass Transfer, Vol. 13, pp.939-942 (1970).

12. Moffat, R. J. and Kays, W. M., "The Turbulent Boundary Layer on a Porous Plate: Experimental Heat Transfer with Uniform Blowing and Suction", Int. Jn. Heat and Mass Transfer, Vol。11, pp. 1547-1566, (1968)。 
13. Whitten, D. G., Moffat, R. Jo, and Kays, W. M., "Heat Transfer to a Turbulent Boundary Layer with Nonuniform Blowing and Surface Temperature", Proceedings of the Fourth International Heat Transfer Conference, Versailles, (Heat Transfer 1970) p. FC8.8.

14. Whitten, D. Go, Kays, W. M., and Moffat, R. J., "The Turbulent Boundary Layer on a Porous Plate: Experimental Heat Transfer with Variable Suction, Blowing, and Surface Temperature", Report No. HMT-3, Thermosciences Div., Dept. of Mechanical Engineering, Stanford University, Stanford Cal., Dec. 1967.

15. Kays, W. Mo, Ccnvective Heat and Mass Trarsfer, MeGrar U:II Book Co., New York, $(1966)$.

16. Thielbahr, W. H., Kays, W。 M., and Moffat, R. J., "The Turbulent Boundary Layer: Experimental Heat Transfer with Blowing, Suction, and Favorable Pressure Gradient", Report No. HMT-5, Thermosciences Div., Dept. of Mechanical Engineering, Stanford University, Stanford, California, Apr. 1969.

17. Van Driest, E. R., "On Turbulent Flow Near a Wal1", J。Aero. Sci., Vol. 23, p. 1007 (1956).

18. Simpson, R. L., "Characteristics of Turbulent Boundary Layers at Low Reynolds Numbers with and without Transpiration", J.Fluid Mech. Vol. 42, Part 4, pp. 769-8c2 (1970).

19. Julien, H. Lo, Kays, W. Mo, and Moffat, R. J., "The Turbulent Boundary Layer on a Porous Plate: Experimental Study of the Effects of a Favorable Pressure Gradient", Report No. HMT-4, Thermosciences Div。, Dept. of Mechanical Engineering, Stanford University, Stanford, Cal., Apr. 1969.

20. Loyd, R. J•, Moffat, R. J., and Kays, W॰ M•, "The Turbulent Boundary Layer on a Porous Plate: An Experimental Study of the Fluid Dynamics with Strong Favorable Pressure Gradients and Blowing", Report No. HMT-13, Thermosciences Div., Dept. of Mechanical Engineering, Stanford University, Stanford, Cal., May 1970.

21. Evans, H. L., Lea, J. F., and Swayne, D. A., "The Couette-Flow ModeI in Turbulent Boundary Layers", Research Report No. 16, Department of Mechanical Engineering, University of Waterloo, Waterloo, Ontario, Feb. 1970.

22. Anderson, P. So, Personal Communication.

23. Launder, B., and Jones, W. P., "On the Prediction of Laminarization", Paper presented at a meeting of the ARC Heat and Mass Transfer Subcomittee, Apr. 5, 1968.

24. Simpson, R. L., Whitten, D. G., Moffat, R. J., "An Experimental Study of the Turbulent Prandtl Number of Air with Injection and Suction", Int. Jn. Heat and Mass Transfer, Vo1. 13, pp. 125-143 (1970). 
25. Kearney, D. W., Moffat, R. Jo, and Kays, W. M., "The Turbulent Boundary Layer: Experimental Heat Transfer with Strong Favorable Pressure Gradients and Blowing", Report No. HMT-12, Thermosciences Div., Department of Mechanical Engineering, Stanford University, Stanford, California, Apr. 1970.

26. Jenkins, R., "Variation of the Eddy Conductivity with Prandtl Modulus and its Use in Prediction of Turbulent Heat Transfer Coefficients", Transactions of Heat Transfer and Fluid Mechanics Institute, pp. 147-158 (1951)。

27. Spalding, D. B., and Patankar, S. V., Heat and Mass Transfer in Boundary Layers, Morgan-Grampian: London (1967). 


\section{Acknowledgements}

The research work upon which this paper is based was made possible by grants from the National Aeronautics and Space Administration, and from the National Science Foundation. The author would particularly like to express appreciation to Mr. Robert Graham of NASA, Lewis Research Center, and to Dr. Royal Rostenbach of NSF.

A very special acknowledgement is also due to Professor $R$. J. Moffat of Stanford University, without whose genius for careful and innovative experiment design and laboratory measurement, this project would never have been possible. 


\section{Figure Captions}

Fig. 1 Diagram of the Boundary Layer System Considered

Fig. 2 Friction Coefficients for the Case of Constant Free-Stream VeIocity With Various Values of Constant Blowing Fraction F, and Constant Blowing Parameter $B_{f}$

Fig. 3 Ratio of Friction Coefficient to Friction Coefficient Without Transpiration but at the same Momentum Thickness Reynolds Number, Plotted as a Function of a Modified Blowing Parameter $b_{f}$.Comparison of Turbulent Experiments With the Exact Laminar Similarity Solution Results

Fig. 4 Shape Factor Plotted as a Function of Blowing Fraction for Various Values of Momentum Thickness Reynolds Number, Free-Stream Velocity Constant

Fig. 5 Stanton Number Plotted as a Function of Enthalpy Thickness Reynolds Number for Various Constant Values of Blowing Fraction, and Various Constant Values of Blowing Parameter for a Fluid With $\mathrm{Pr}_{\mathrm{r}}=0.7$ and Constant Free-Stream Velocity

Fig. 6 The Ratio of Stanton Number to Stanton Number for No Blowing at the Same Value of Enthalpy Thickness Reynolds Number Plotted as a Function of Modified Blowing Parameter. Comparison of the Experimental Results With the Exact Laminar Similarity Solutions, and the Equation Proposed by Kutateladze and Leont'ev.

Fig. 7 The Effect of a Step-Down in Blowing on the Stanton Number, Constant Free-Stream Velocity

Fig. 8 The Effect of a Step-Up in Blowing on the Stanton Number, Constant Free-Stream Velocity

Fig. 9 The Effects of a Combination of Blowing and Acceleration on Stanton Number

Fig. 10 Values of Effective Sublayer Thicknesses $\mathrm{A}^{+}$and $\mathrm{B}^{+}$for the Case of No Transpiration and No Pressure Gradient Plotted as a Function of Mixing-Length Constant, $\mathrm{k}$. The Indicated Combinations of $\mathrm{A}^{+}$or $\mathrm{B}^{+}$ and $\mathrm{k}$ will Predict Identical Values of $\mathrm{u}^{+}$at $\mathrm{y}^{+}=80$.

Fig. 11 The Effect of Pressure Gradient and Transpiration on $A^{+}$, Curves Generated from an Empirical Fit to Available Data

Fig. 12 The Effect of Pressure Gradient and Transpiration on $\mathrm{B}^{+}$, Using the Same Basic Data as for Fig. 11 
Fig. 13 Examples of the Use of the $\mathrm{B}^{+}$Scheme and a Proposed Turbulent Prandt 1 Number Function to Predict the Velocity and Temperature Laws-of-the-Wall

Fig. 14 Comparison of Predictions and Experiment for the Velocity Lawmof the-Wal1 for Two Cases of Transpiration and One-Case of Both Transpiration and Pressure Gradient

Fig. 15 Turbulent Prandt1 Number as Determined from Velocity and Temperature Profiles for Various Blowing and Suction Fractions, Constant FreeStream Velocity

Fig. 16 Turbulent Prandt 1 Numbers Determined from Velocity and Temperature Profiles for a Wide Range of Strong Accelerations With Various Values of Blowing

Fig. 17 Turbulent Prandt1 Numbers Determined from Experimental Temperature Profiles, Together With Predicted Velocity Profiles Using the $\mathrm{B}^{\frac{t^{t}}{}}$ Scheme

Fig. 18 An Example of a Prediction of Friction and Heat Transfer For a Case of Very Strong Blowing and Moderately Strong Acceleration, Followed by Constant Free-Stream Velocity

Fig. 19 Comparison of Experimental and Predicted Velocity Profiles for the Same Case as Considered in Fig. 18, One Profile Near the End of the Accelerated Region, and One Profile In the Downstream Recovery Region

Fig. 20 Comparison of Predicted and Experimenta1 Temperature Profiles under the Same Conditions as Described Under Fig. 19

Fig. 21 Comparison of Predicted and Experimental Stanton Numbers for a Flow Starting With Constant Free-Stream Velocity and Constant Blowings Followed by Very Strong Acceleration. In the Middle of the Accelerated Region the Blowing is Shut off Abruptly. Finally the Acceleration is Shut Off so that the Last Section is at Zero Blowing and Constant Free-Stream Velocity. 


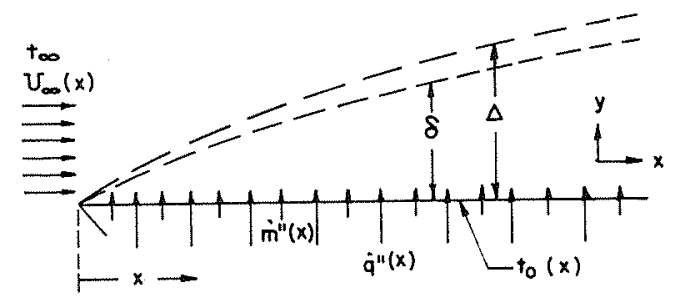

FIG. I

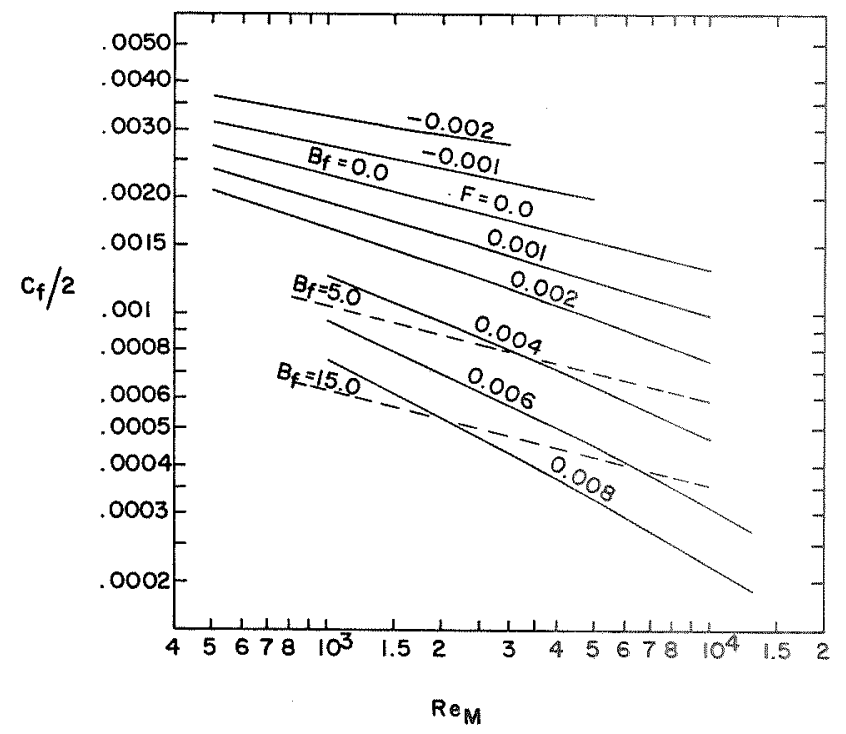

FIG. 2

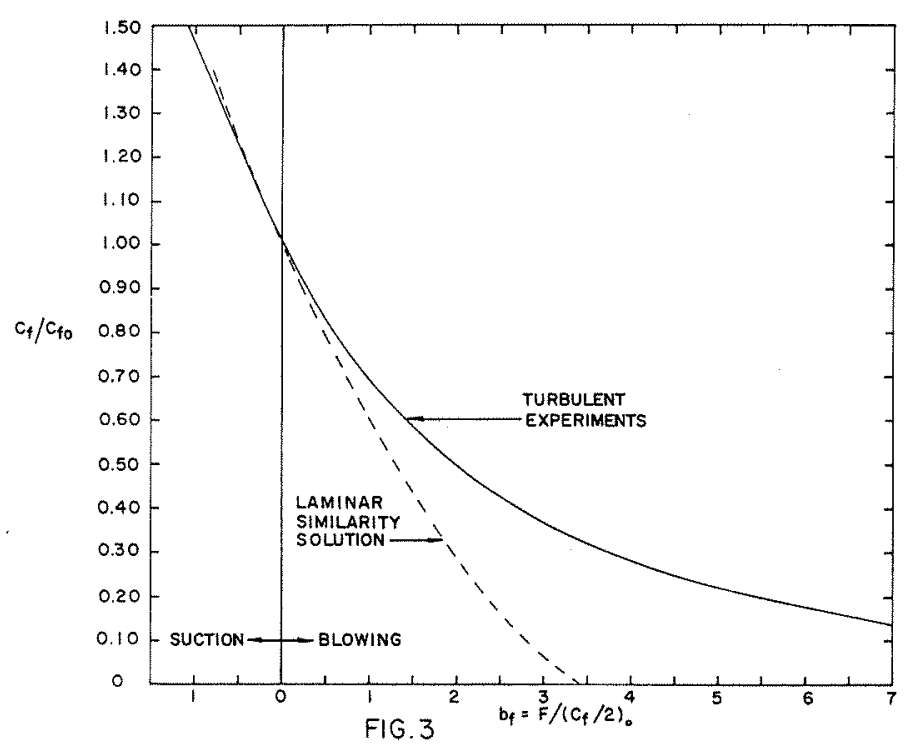

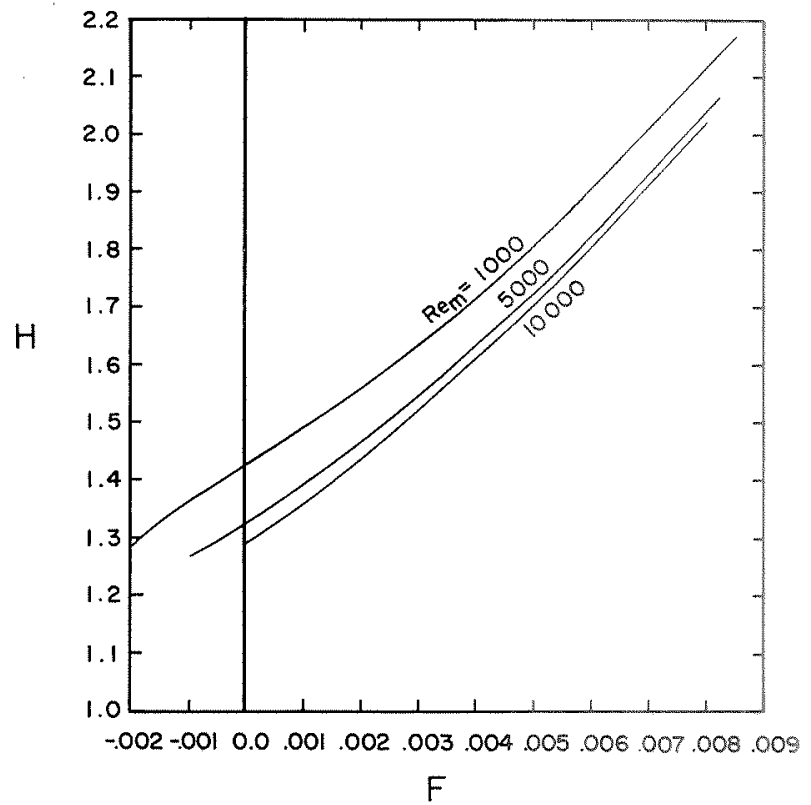

FIG 4 


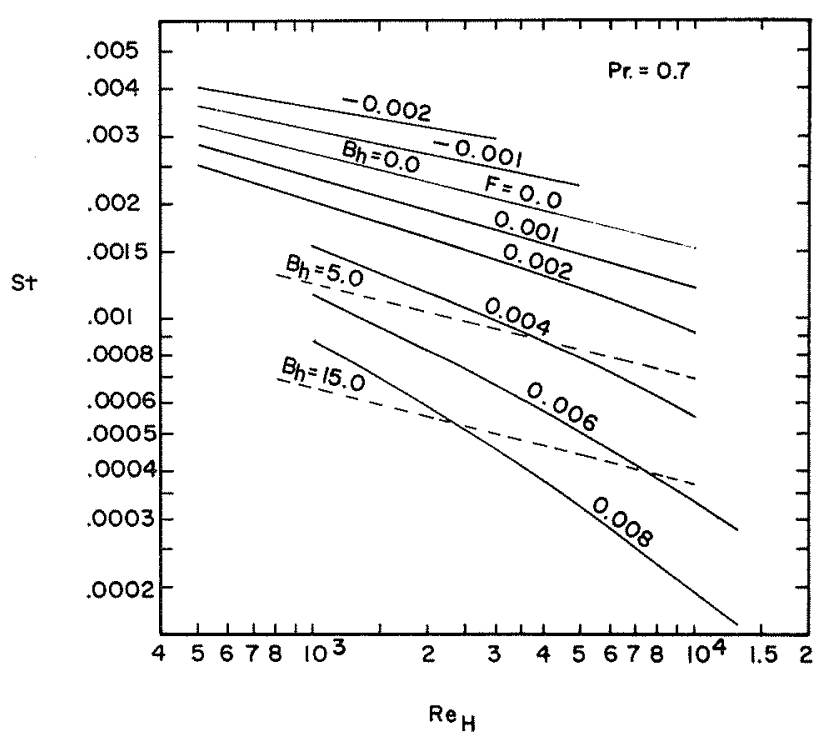

FIG. 5

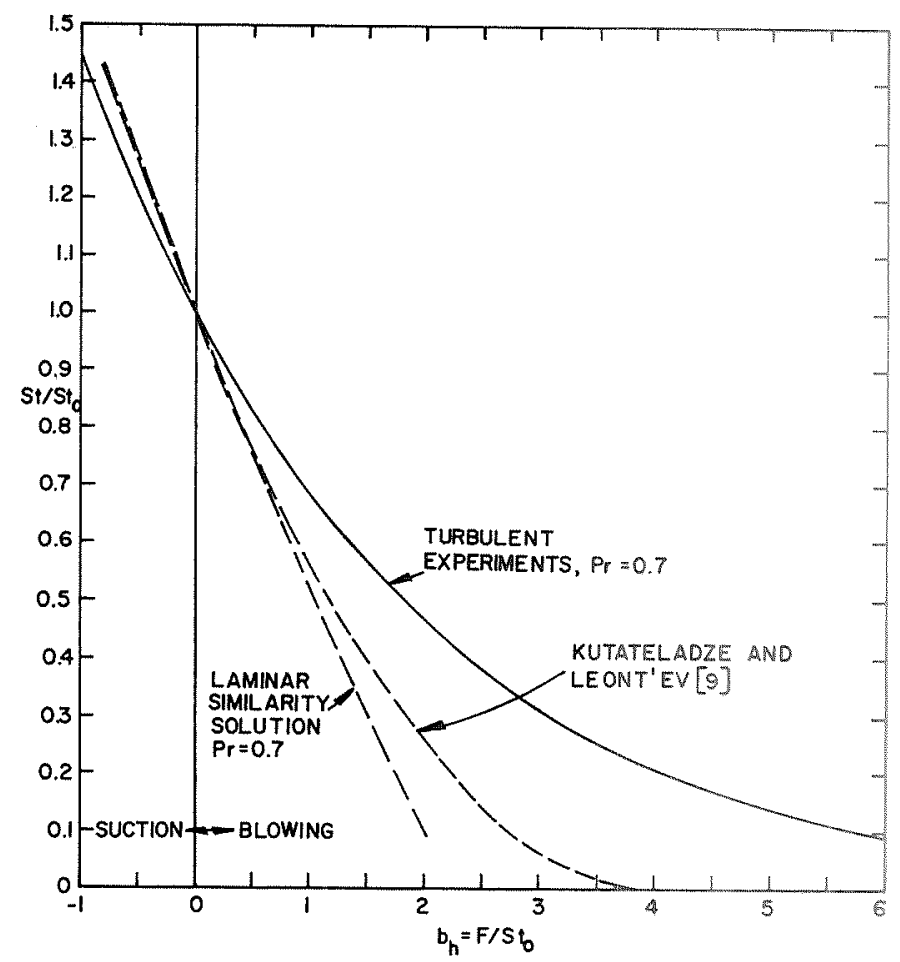

FIG. 6

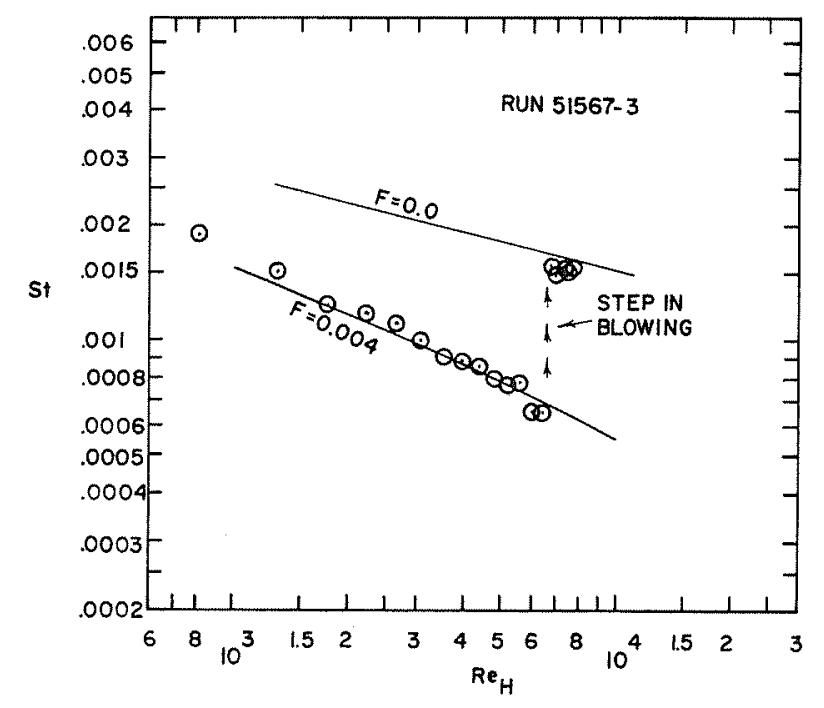

FIG. 7

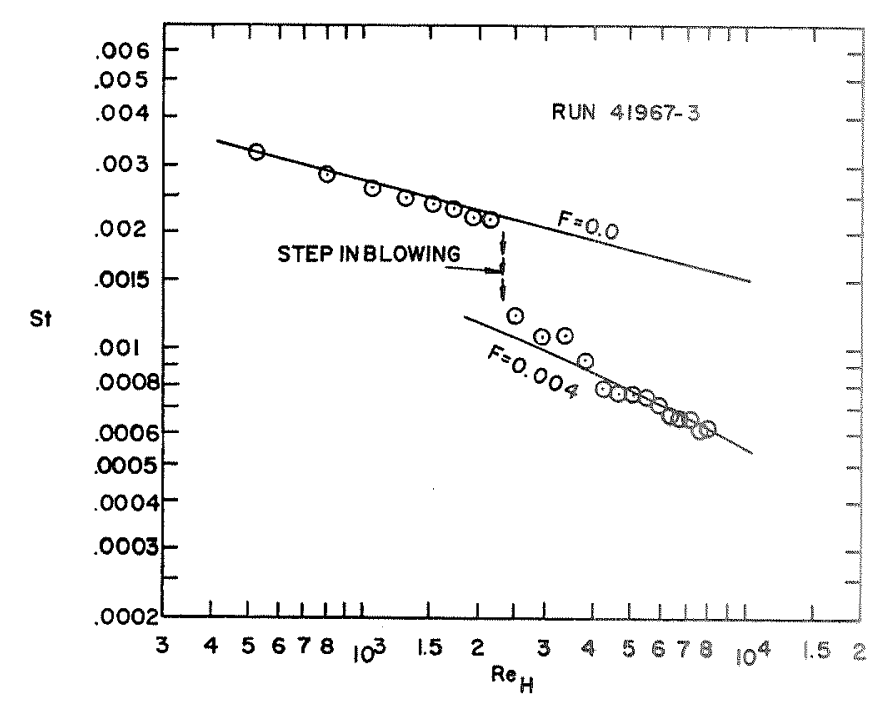

FIG. 8 


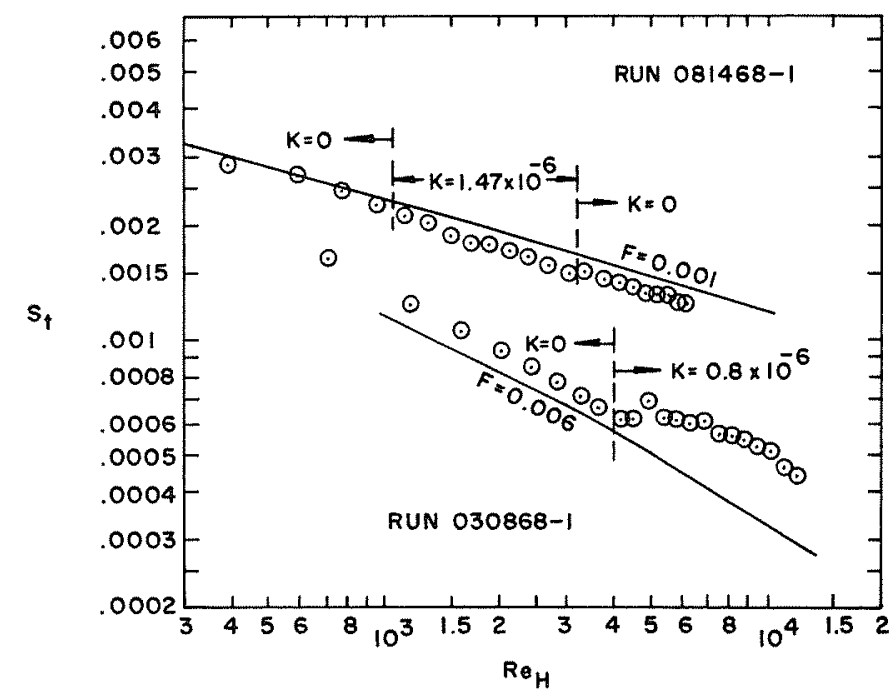

FIG. 9

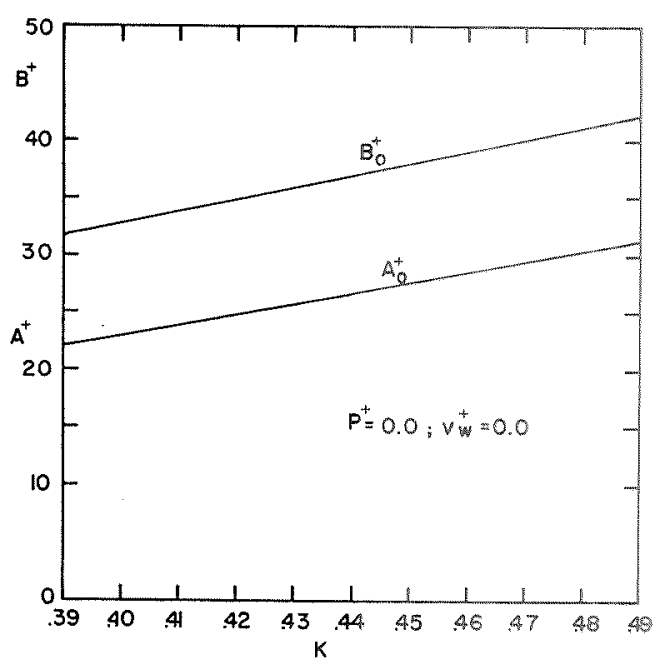

FIG.IO

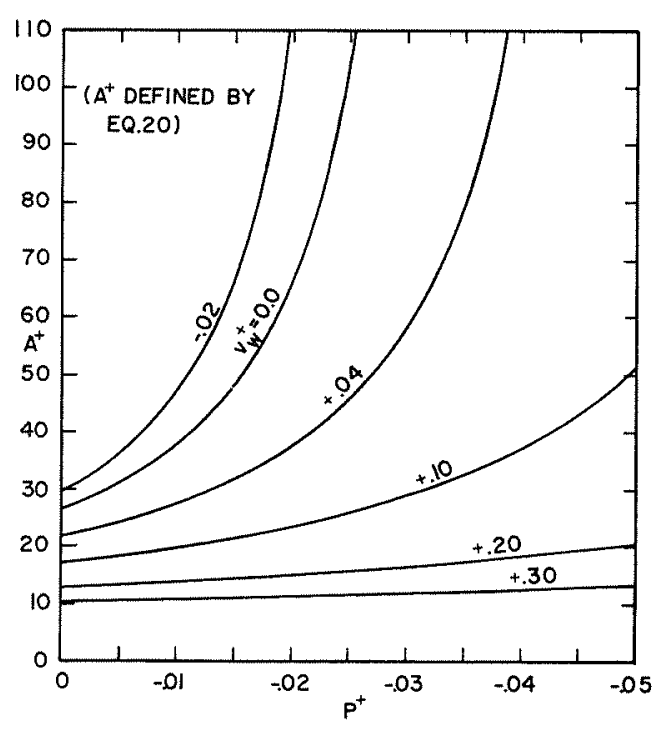

FIG.II

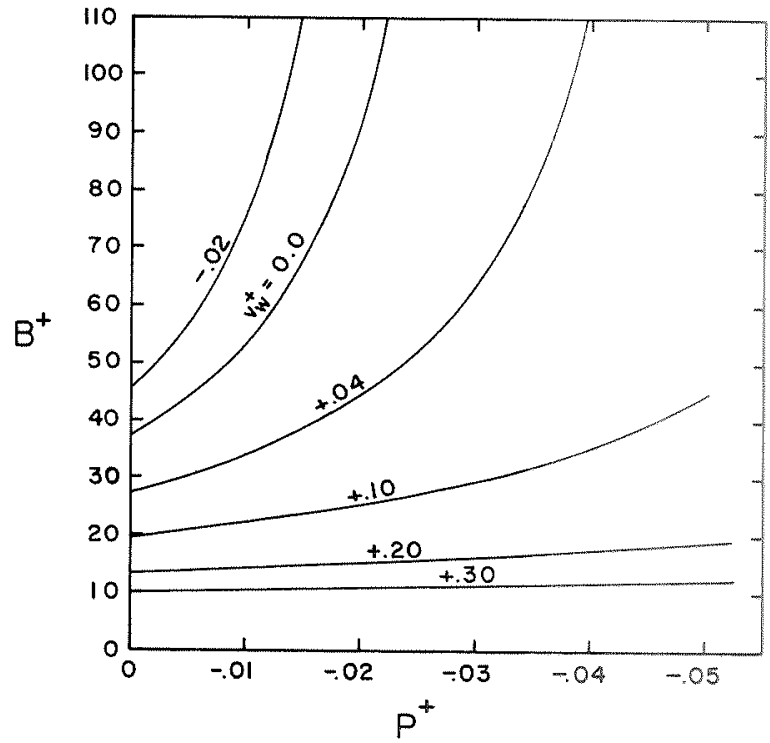

FIG. 12 


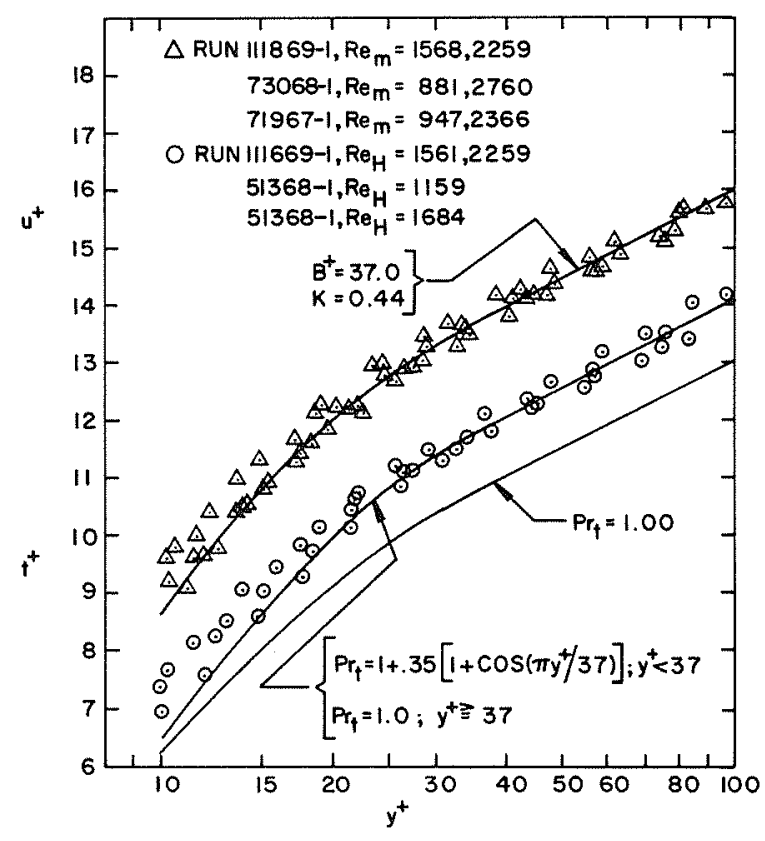

FIG. 13

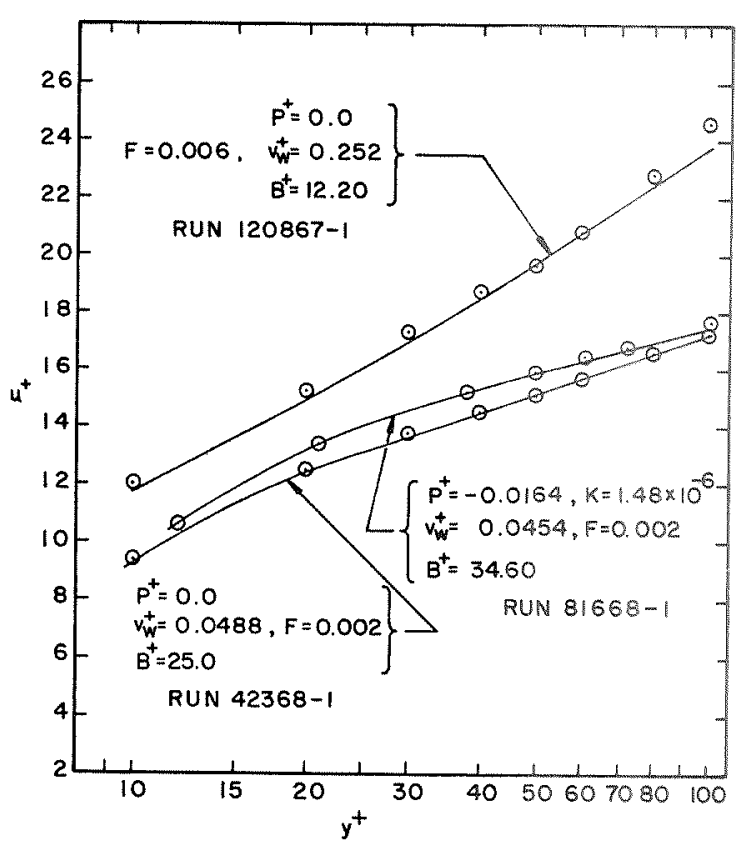

FIG. 14

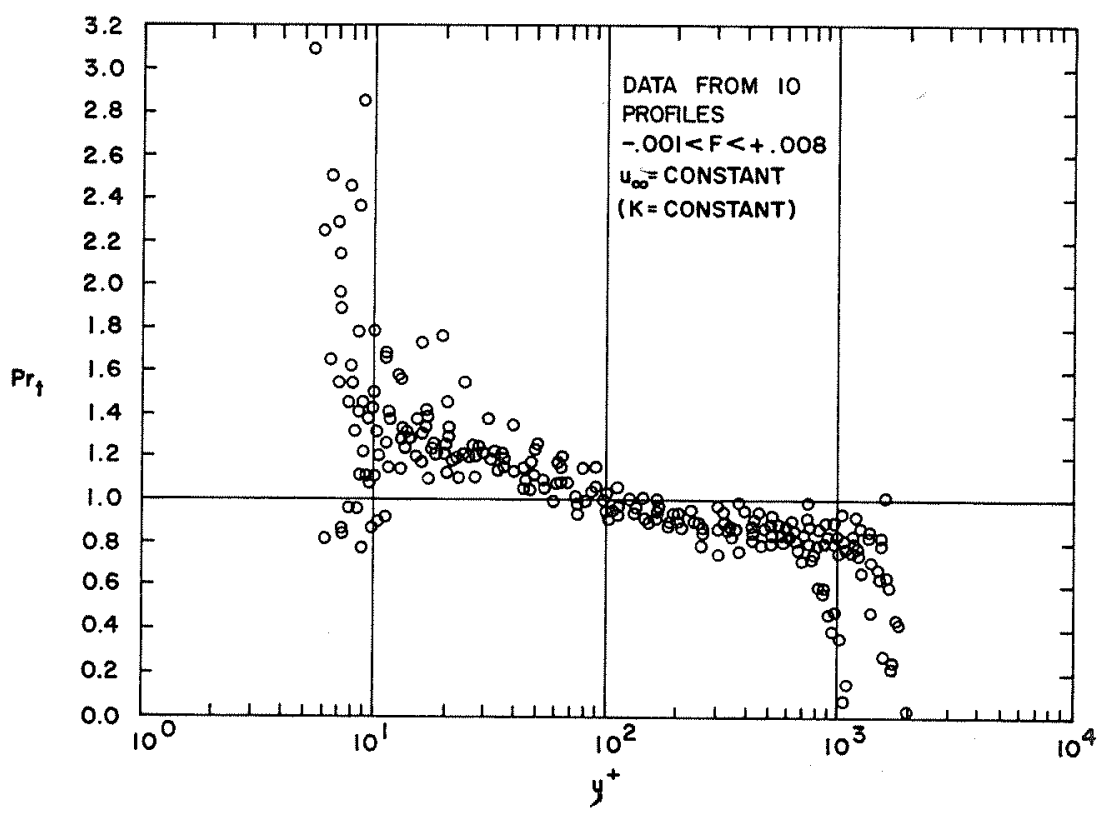

FIG. 15 


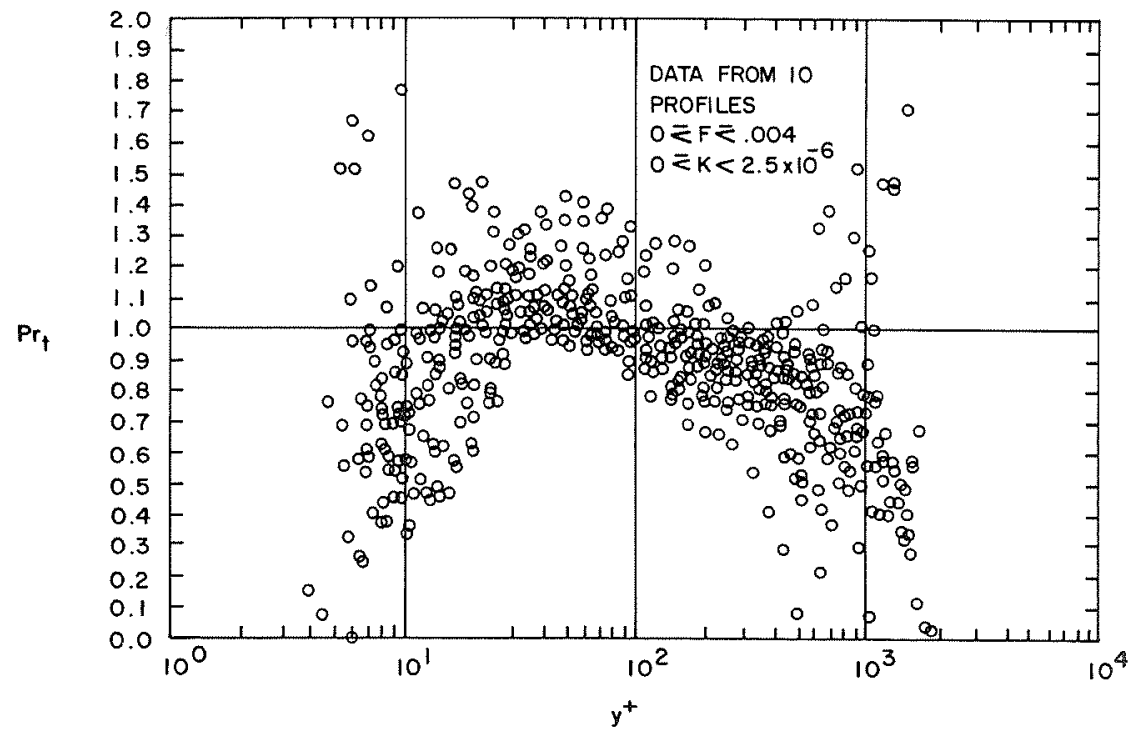

FIG. 16

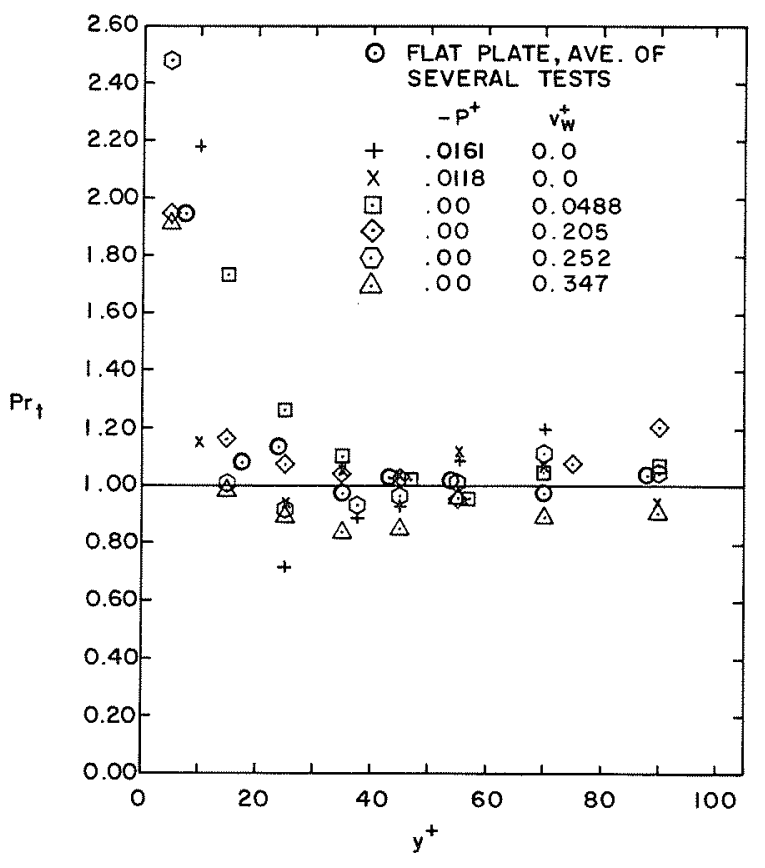

FIG.17

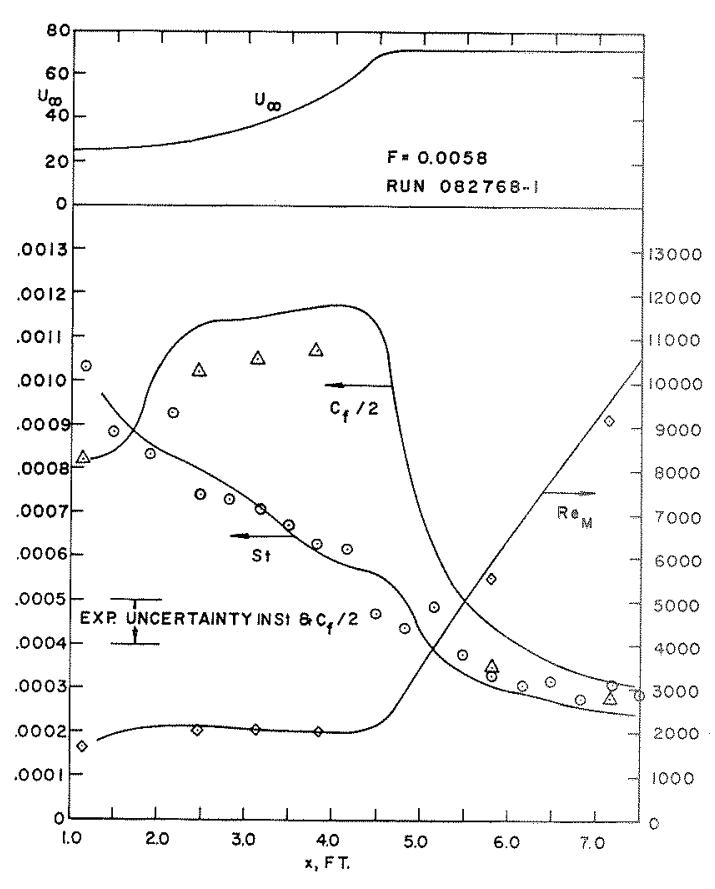

FIG.18 


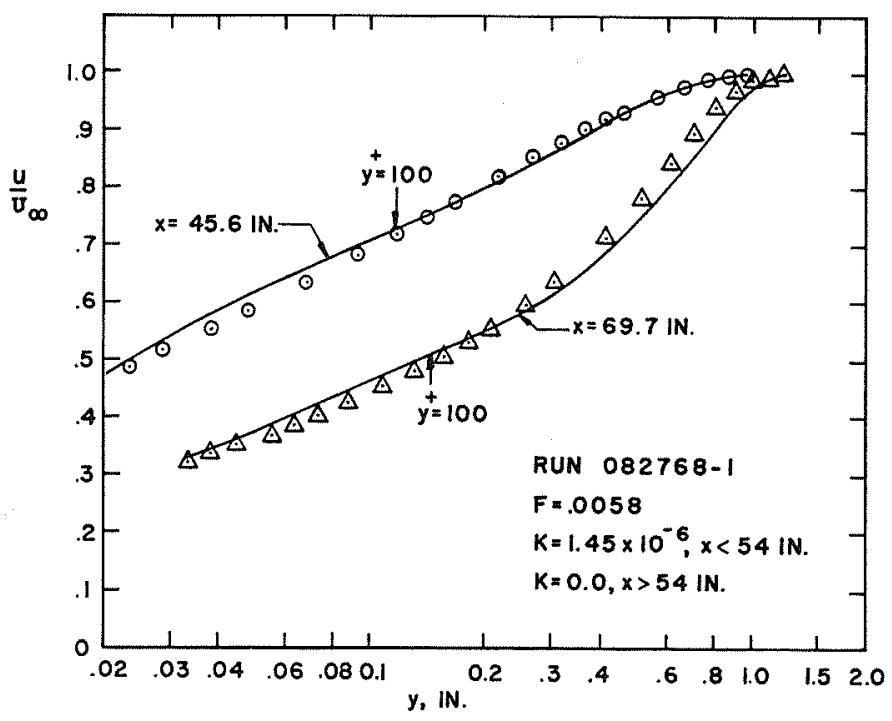

FIG. 19

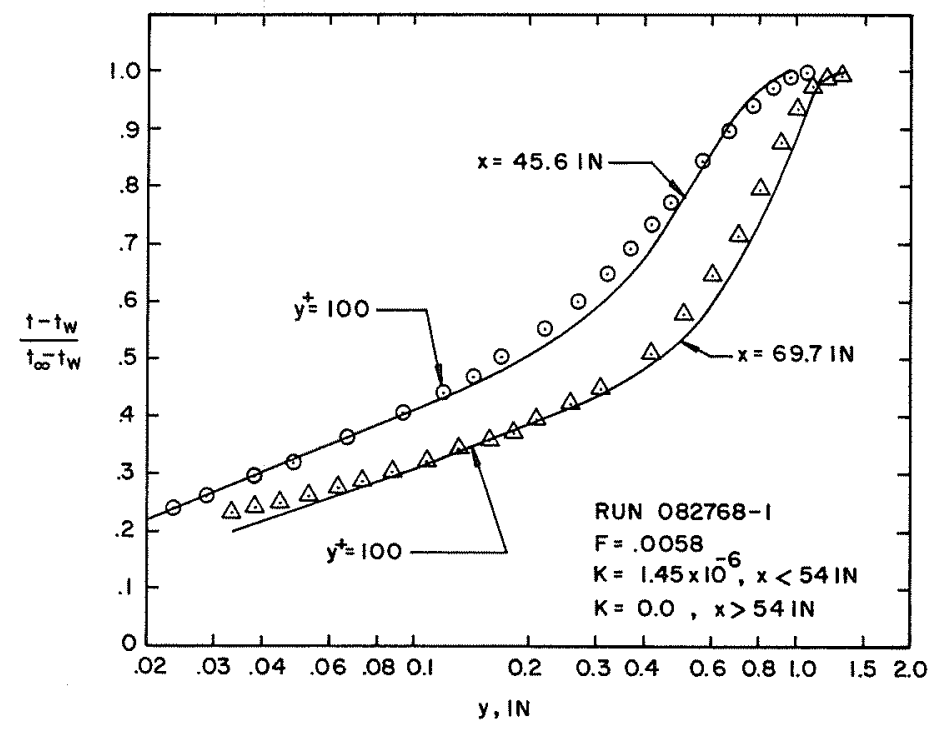

FIG. 20

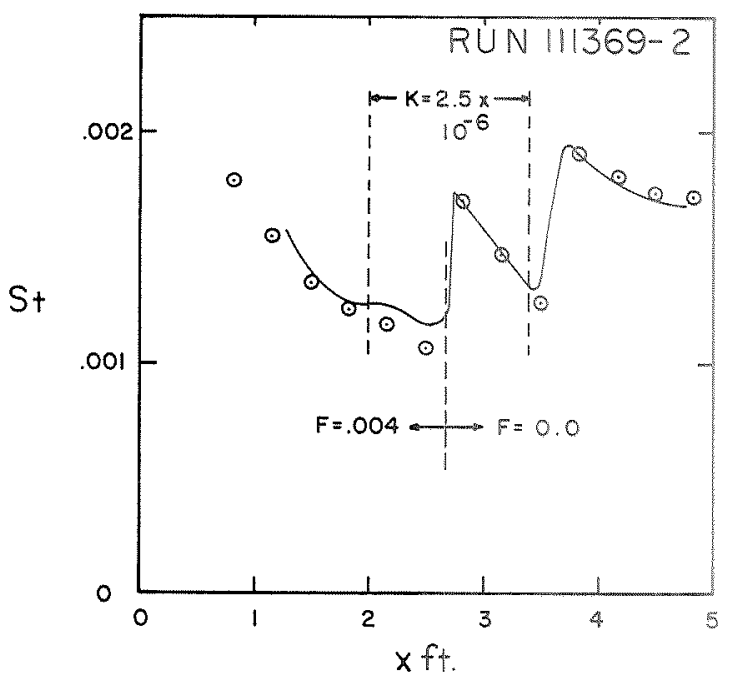

FIG. 21 